\title{
THE COMPOSITION AND DISTRIBUTION OF DUST ALONG THE LINE OF SIGHT TOWARD THE GALACTIC CENTER ${ }^{1}$
}

\author{
J. E. Chiar, ${ }^{2}$ A. G. G. M. Tielens, ${ }^{3}$ D. C. B. Whittet, ${ }^{4}$ W. A. Schutte, ${ }^{5}$ A. C. A. Boogert, ${ }^{3,6}$ D. Lutz, ${ }^{7}$ \\ E. F. VAN DishoeCK, ${ }^{5}$ AND M. P. BERNSTEIN ${ }^{8}$ \\ Received 1999 July 30; accepted 2000 February 18
}

\begin{abstract}
We discuss the composition of dust and ice along the line of sight to the Galactic center (GC) based on analysis of mid-infrared spectra $(2.4-13 \mu \mathrm{m})$ from the Short Wavelength Spectrometer on the Infrared Space Observatory (ISO). We have analyzed dust absorption features arising in the molecular cloud material and the diffuse interstellar medium along the lines of sight toward Sgr $\mathrm{A}^{*}$ and the Quintuplet sources, GCS 3 and GCS 4. It is evident from the depth of the $3.0 \mu \mathrm{m} \mathrm{H}_{2} \mathrm{O}$ and the $4.27 \mu \mathrm{m} \mathrm{CO} \mathrm{CO}_{2}$ ice features that there is more molecular cloud material along the line of sight toward Sgr $\mathrm{A}^{*}$ than toward GCS 3 and GCS 4. In fact, Sgr A* has a rich infrared ice spectrum with evidence for the presence of solid $\mathrm{CH}_{4}, \mathrm{NH}_{3}$, and possibly $\mathrm{HCOOH}$. Hydrocarbon dust in the diffuse interstellar medium along the line of sight to the GC is characterized by absorption features centered at 3.4, 6.85, and $7.25 \mu \mathrm{m}$. Ground-based studies have identified the $3.4 \mu \mathrm{m}$ feature with aliphatic hydrocarbons, and ISO has given us the first meaningful observations of the corresponding modes at longer wavelengths. The integrated strengths of these three features suggest that hydrogenated amorphous carbon is their carrier. We attribute an absorption feature centered at $3.28 \mu \mathrm{m}$ in the GCS 3 spectrum to the $\mathrm{C}-\mathrm{H}$ stretch in aromatic hydrocarbons. This feature is not detected, and its $\mathrm{C}-\mathrm{C}$ stretch counterpart appears to be weaker, in the Sgr A* spectrum. A key question now is whether or not aromatics are a widespread component of the diffuse interstellar medium, analogous to aliphatic hydrocarbons.
\end{abstract}

Subject headings: dust, extinction - Galaxy: center - infrared: ISM: lines and bands infrared: stars - ISM: molecules

\section{INTRODUCTION}

Bright infrared (IR) sources combined with $30 \mathrm{mag}$ of visual extinction have made the Galactic center (GC) an attractive line of sight for the study of interstellar dust composition. Absorption features in the spectra of GC IR sources have been used extensively to characterize interstellar grain material, including silicates (Becklin et al. 1978; Roche \& Aitken 1985) and hydrocarbons (Willner et al. 1979; McFadzean et al. 1989; Sandford et al. 1991; Pendleton et al. 1994). Previous observations of the GC suggested the presence of absorptions expected in icy grain material, most notably the $3 \mu \mathrm{m}$ band of $\mathrm{H}_{2} \mathrm{O}$ ice (e.g., McFadzean et al. 1989) but, until recently, the explanation that one or more molecular clouds obscure the GC and contribute to its total extinction had not been completely accepted since the $3 \mu \mathrm{m}$ feature has a substantially different

\footnotetext{
${ }^{1}$ Based on observations made with the Infrared Space Observatory, an ESA project with instruments funded by ESA member states (especially the PI countries: France, Germany, the Netherlands, and the United Kingdom) and with the participation of ISAS and NASA.

${ }^{2}$ NASA/Ames Research Center, Mail Stop 245-3, Moffett Field, CA 94035; and SETI Institute, Mountain View, CA 94043; chiar@misty.arc.nasa.gov.

${ }^{3}$ Kapteyn Astronomical Institute, P.O. Box 800, 9700 AV Groningen, Netherlands.

${ }^{4}$ Rensselaer Polytechnic Institute, Department of Physics, Applied Physics, and Astronomy, Troy, NY 12180.

${ }^{5}$ Leiden Observatory, P.O. Box 9513, 2300 RA Leiden, Netherlands.

${ }^{6}$ Present address: California Institute of Technology, Downs Laboratory of Physics, Mail Code 320-47, Pasadena, CA 91125.

${ }_{7}$ Max-Planck-Institut für extraterrestrische Physik, Postfach 1603, 85740 Garching, Germany.

${ }^{8}$ NASA/Ames Research Center, Mail Stop 245-6, Moffett Field, CA 94035 and SETI Institute, Mountain View, CA 94043.
}

profile than that observed in dense cloud sources (Tielens et al. 1996; Wada, Sakata, \& Tokunaga 1991). The presence of molecular cloud material in the GC was confirmed via detection of solid $\mathrm{CO}_{2}$ features by the Short Wavelength Spectrometer (SWS) on the Infrared Space Observatory (ISO) (Gerakines et al. 1999; de Graauw et al. 1996b; Lutz et al. 1996).

Solid organic material is a well-established component of the diffuse interstellar medium. The complex $3.4 \mu \mathrm{m}$ feature, ubiquitous along sight lines that sample the diffuse interstellar medium (ISM) in our Galaxy and other galaxies (e.g., Pendleton et al. 1994; Bridger, Wright, \& Geballe 1993), is identified with short-chained aliphatic hydrocarbons with a methylene/methyl $\left(-\mathrm{CH}_{2}-/-\mathrm{CH}_{3}\right)$ group ratio between 2 and 2.5 (Sandford et al. 1991; Pendleton et al. 1994). The $\mathrm{CH}$ deformation mode at $6.8 \mu \mathrm{m}$ was detected in Kuiper Airborne Observatory data (Tielens et al. 1996), although the weaker $7.3 \mu \mathrm{m}$ band could not be confirmed at the time. One model proposes that organic refractory matter in the diffuse ISM is the product of energetic processing of simple ices (such as $\mathrm{CO}, \mathrm{H}_{2} \mathrm{O}, \mathrm{CH}_{3} \mathrm{OH}$ ) in dense clouds (e.g., Allamandola, Sandford, \& Valero 1988; Greenberg et al. 1995; Strazzulla, Castornia, \& Palumbo 1995; Moore, Ferrante, \& Nuth 1996). However, the discovery of spectroscopic similarity between diffuse interstellar and circumstellar organics has prompted a reassessment of the origin of organic matter in the ISM (Lequeux \& Jourdain de Muizon 1990; Chiar et al. 1998b). The striking similarity of the circumstellar and interstellar $3.4 \mu \mathrm{m}$ features indicates that at least some of the organic carriers that lead to the $3.4 \mu \mathrm{m}$ absorption in the diffuse ISM originate as stardust (Chiar et al. 1998b). The case for production of the $3.4 \mu \mathrm{m}$ band carrier in dense clouds and thus its presence in organic refractory mantles is further weakened by the finding of 
Adamson et al. (1999) that, unlike the $9.7 \mu \mathrm{m}$ silicate feature (e.g., Aitken et al. 1986), the $3.4 \mu \mathrm{m}$ feature along the line of sight toward Sgr A* (IRS 7) is not polarized. Hence, the 3.4 $\mu \mathrm{m}$ band is not produced by a carrier residing in a mantle on a silicate core but by very small (unaligned) grains. Nevertheless, energetic processing of ices in dense clouds may still play some role in the production of interstellar organics since diffuse ISM dust cycles into dense clouds fairly rapidly ( $\sim 3 \times 10^{7} \mathrm{yr}$; McKee 1989; Jones et al. 1994).

Hydrogenated amorphous carbon (HAC) is a possible candidate for the organic component of diffuse interstellar dust (Tielens et al. 1996; Pendleton et al. 1994), and several laboratory groups are attempting to produce $\mathrm{HAC}$ analogs with a $-\mathrm{CH}_{2}-/-\mathrm{CH}_{3}$ ratio that matches the observed feature (e.g., Furton, Laiho, \& Witt 1999; Duley et al. 1998). Previous comparisons of HACs with the interstellar $3.4 \mu \mathrm{m}$ feature have shown that many HACs produced in the laboratory are lacking in $-\mathrm{CH}_{3}$ groups relative to $-\mathrm{CH}_{2}-$ groups, thus providing an unsatisfactory fit to the observed feature (e.g., Pendleton et al. 1994). Recently, Duley et al. (1998) have shown that the $3.38 \mu \mathrm{m}-\mathrm{CH}_{3}$ subfeature is strengthened at lower temperatures $(77 \mathrm{~K})$, and the resulting $\mathrm{HAC}$ with $-\mathrm{CH}_{2}-/-\mathrm{CH}_{3}<1$ provides a better match to the $3.4 \mu \mathrm{m}$ interstellar feature in comparison with that of HACs at a higher temperature (e.g., $300 \mathrm{~K}$ ).

Recently, a feature near $6.2 \mu \mathrm{m}$ was discovered along sight lines toward the GC Quintuplet sources and several dusty late-type WC Wolf-Rayet stars and has been attributed to the aromatic $\mathrm{C}-\mathrm{C}$ stretch in polycyclic aromatic hydrocarbon molecules (PAH) in the diffuse ISM along the line of sight (Schutte et al. 1998). PAHs are a certain component of the carbonaceous material in the Galaxy, as shown by the dominance and ubiquitous presence of the family of PAH emission features at 3.3, 6.2, 7.7, 8.6, and 11.3 $\mu \mathrm{m}$ in diffuse emission in the Galactic disk (Giard et al. 1988; Ristorcelli et al. 1994; Mattila et al. 1996). Therefore, the detection of the aromatic $\mathrm{C}-\mathrm{C}$ stretch at $6.2 \mu \mathrm{m}$ in diffuse ISM sight lines is not surprising; its carrier may be in the form of gaseous polycyclic aromatic hydrocarbons (PAHs) or PAH clusters with sizes up to a few thousand C atoms (Schutte et al. 1998). The corresponding $\mathrm{C}-\mathrm{H}$ stretch at $\sim 3.3 \mu \mathrm{m}$ was not detected in absorption in the diffuse ISM, although high signal-to-noise data, necessary to detect this weak feature, were not available for many sight lines. However, an absorption feature at $3.25 \mu \mathrm{m}$ is detected in ground-based data in five heavily extincted molecular cloud sight lines (Sellgren et al. 1995; Brooke, Sellgren, \& Geballe 1999).

This paper discusses the composition of the hydrocarbon dust component, as well as the molecular cloud ices, along the line of sight to the Galactic center based on 2.5-13 $\mu \mathrm{m}$ spectra from the Infrared Space Observatory's Short Wavelength Spectrometer (ISO-SWS). ISO-SWS has revealed the previously elusive $2.95 \mu \mathrm{m} \mathrm{NH}$ feature in dense cloud material along the GC line of sight. These observations also have provided us with the first meaningful observations of hydrocarbon modes at long wavelengths $(5-8 \mu \mathrm{m})$ corresponding to the well-studied $3.4 \mu \mathrm{m}$ absorption feature, associated with the diffuse ISM, allowing us to narrow down the list of possible hydrocarbon candidates (§ 5.1). We also present the first detection of aromatic absorption, at $3.28 \mu \mathrm{m}$, unassociated with molecular cloud dust. This paper is organized as follows. In $\S 2$ we briefly discuss what is known about the nature of the sources, and in $\S 3$ we explain the data reduction, and present the ISO-SWS spectra of three sources in the Galactic center. In the following sections, we discuss the nature of the molecular cloud IR absorption features $(\S 4)$ and hydrocarbon absorption features $(\S 5)$ along the line of sight. Finally, we discuss possible scenarios for the origin of hydrocarbons in the GC region $(\S 6)$ and summarize our results $(\S 7)$.

\section{THE NATURE OF THE SOURCES}

This paper analyzes spectra from ISO-SWS for three sight lines toward the Galactic center: Sgr A* (and Sgr A W-IRS 3), GCS 3, and GCS 4 . The IR sources contained in the ISO beam along the line of sight toward Sgr A* and Sgr A W-IRS 3 suffer visual extinction of $\sim 31 \mathrm{mag}$ (Rieke, Rieke, \& Paul 1989 and references therein) and contain M giants, supergiants, and $\mathrm{H}$ II regions (Eckart et al. 1995; Sellgren et al. 1987; Lebofsky, Rieke, \& Tokunaga 1982; Wollman, Smith, \& Larson 1982). GCS 3 and GCS 4, located near the GC Radio Arc, approximately 14' northeast of the Galactic center and corresponding to a projected distance of about $30 \mathrm{pc}$ (assuming a distance of $8.2 \mathrm{kpc}$ ), were first discovered in the polarimetric study by Kobayashi et al. (1983). Visual extinction estimates are similar to that for the GC, $A_{V} \sim 29 \mathrm{mag}$ (Figer, McLean, \& Morris 1999 and references therein). Detailed imaging of the region revealed that GCS 3 and GCS 4 consist of a compact group of at least five IR sources, which have since become known as the Infrared Quintuplet (Nagata et al. 1990; Okuda et al. 1990), although more recent imaging and spectroscopic investigations (Moneti, Glass, \& Moorwood 1992, 1994) have shown that this region contains several bright and many faint IR sources. The nature of these objects is still open to debate: several studies have suggested that the Quintuplet cluster consists of young stars surrounded by thick shells or cocoons of hot dust (e.g., Moneti et al. 1994). A very recent photometric study has proposed that these stars are actually massive late-type dusty WC Wolf-Rayet stars (Figer, McLean, \& Morris 1999).

\section{OBSERVATIONS AND DATA REDUCTION}

Infrared spectra of Sgr A* (Lutz et al. 1996), Sgr A W-IRS 3 , and Quintuplet (RAFGL 2004) sources GCS 3 and GCS 4 were obtained with ISO-SWS in 1996 and 1997. The reader is referred to de Graauw et al. (1996a) for a more detailed description of SWS and its capabilities. Observational details and coordinates are listed in Table 1 . The ISO-SWS $14^{\prime \prime} \times 20^{\prime \prime}$ beam was centered on the coordinates listed in Table 1; the Sgr A* and Sgr A W-IRS 3 positions are only several arcseconds apart and therefore probe similar lines of sight in the relatively large ISO beam. The observations of GCS 3 were centered on GCS 3 I and included all four GCS 3 objects; GCS 4, located some 15" away from GCS 3 I probes a unique sight line in this region. Data for GCS 3 and GCS 4 have been presented in Schutte et al. 1998; we have re-reduced these data using the most up-to-date calibration files (see below). Astronomical Observing Template 1 (AOT1), speed 4, was used to obtain data for Sgr A* in the 2-45 $\mu$ m spectral region (Lutz et al. 1996), which gives a resolving power of $R \sim 800-1700$ depending on the wavelength region. AOT1 speed 3 data were obtained for the GCS 3 and GCS 4 in the full 2-45 $\mu \mathrm{m}$ spectral region with $R \sim 480-1020$. Higher resolution data were obtained for GCS 3 with AOT6 in the 3.1-3.5 
TABLE 1

ObSERVATIONAL DetaILS

\begin{tabular}{|c|c|c|c|c|c|c|}
\hline \multirow[b]{2}{*}{ SOURCE } & \multirow[b]{2}{*}{ OTHER ID } & \multicolumn{2}{|c|}{ Position (J2000) } & \multirow[b]{2}{*}{$\mathrm{AOT}^{\mathrm{a}}$} & \multirow[b]{2}{*}{ UTC DATE } & \multirow{2}{*}{$\begin{array}{l}t_{\text {int }} \\
\text { (s) }\end{array}$} \\
\hline & & R. A. & Decl. & & & \\
\hline Sgr A* & - & 174540.0 & -290029 & 1.4 & 1996 Feb 19 & 6528 \\
\hline Sgr A-W IRS $3 \ldots \ldots$ & $\ldots$ & 174539.6 & -290023 & 6 & 1997 Mar 14 & 1086 \\
\hline \multirow[t]{2}{*}{ GCS $3-\mathrm{I} \ldots \ldots \ldots \ldots$} & MGM 5-4 & 174614.9 & -284934 & 1.3 & 1996 Aug 29 & 3454 \\
\hline & & & & 6 & 1996 Oct 8 & 3226 \\
\hline GCS $4 \ldots \ldots \ldots \ldots \ldots$ & MGM 5-3 & 174615.6 & -284947 & 1.3 & 1996 Sept 9 & 3454 \\
\hline
\end{tabular}

NotE.-Units of right ascension are hours, minutes, and seconds, and units of declination are degrees, arcminutes, and arcseconds.

${ }^{\text {a }}$ AOT 1.m represents Astronomical Observing Template 1, speed $m$.

${ }^{b}$ MGM identification from survey by Moneti et al. 1992.

$(R \sim 2000)$ and $5.3-7.0 \mu \mathrm{m}(R \sim 1350)$ spectral regions and for Sgr A W-IRS 3 in the 7.4-8.0 $\mu \mathrm{m}(R \sim 1700)$ spectral region.

This paper discusses the $2.5-13 \mu \mathrm{m}$ spectral region, which contains the strongest absorption features of hydrocarbons and ices excepting the $15.3 \mu \mathrm{m} \mathrm{CO}$ band, which has been discussed extensively by Gerakines et al. (1999 and references therein). The data were reduced using the SWS Interactive Analysis package (de Graauw et al. 1996a) and the Observers SWS Interactive Analysis Package (OSIA). Version 7 of the SWS calibration files were used. The fluxcalibrated spectra from 2.5 to $13 \mu \mathrm{m}$ are shown in Figure 1 .

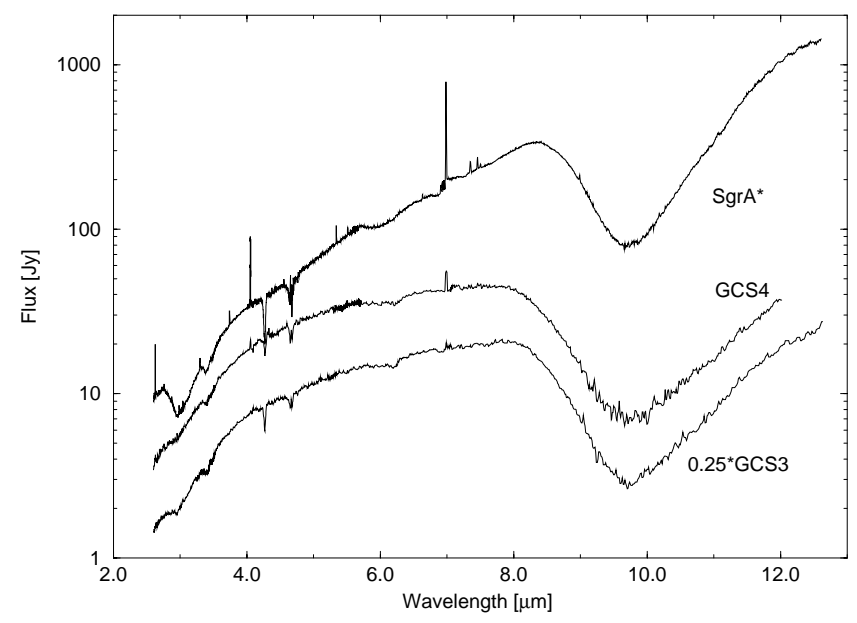

FIG. 1.-ISO-SWS spectra from 2.5 to $13 \mu \mathrm{m}$ of Sgr A* (AOT6), GCS 4 (AOT1), and GCS 3 (AOT1). The GCS 3 spectrum has been divided by 4 for presentation purposes.
Optical depth spectra were determined by fitting local loworder polynomial continua as described in the following sections. No evidence for broad molecular emission features (e.g., caused by PAHs) was apparent in the spectra, thus continua were chosen assuming only the presence of absorption. Optical depths of the dust-absorption features are listed in Table 2.

\section{THE MOLECULAR CLOUD FEATURES}

Molecular cloud absorption features of solid state $\mathrm{H}_{2} \mathrm{O}$, $\mathrm{CO}_{2}$, and $\mathrm{CH}_{4}$ along the line of sight to the Galactic center are assigned based on their previous identification in known molecular cloud sources. Consistent with the uneven distribution of molecular cloud material, these features vary in depth across the GC field, whereas absorption features caused by dust in the diffuse interstellar medium are observed at approximately constant depth (§ 5.1). It is evident from the depth of the $3.0 \mu \mathrm{m}$ ice feature (Fig. 1) that there is more molecular cloud material along the line of sight toward Sgr A* than toward GCS 3 and GCS 4. Thus, we use the Sgr A* spectra to characterize the molecular cloud dust that is present along the line of sight to the Galactic center. Optical depth plots of the molecular cloud features are presented in Figure 2; column densities are listed in Table 3. We discuss these features in detail below.

\section{1. $\mathrm{H}_{2} \mathrm{O}$ Ice at 3.0 and $6.0 \mu \mathrm{m}$}

\subsubsection{The $3 \mu \mathrm{m}$ Feature}

The $3 \mu \mathrm{m}$ optical depth profiles for the GC sources (Fig. 3) were determined by fitting a local second-degree polynomial in the $2.5-4.2 \mu \mathrm{m}$ region. These profiles are very similar to each other and at the same time distinctly differ-

TABLE 2

OPTICAL DEPTHS ${ }^{\mathbf{a}}$

\begin{tabular}{|c|c|c|c|c|c|c|c|c|c|c|}
\hline \multirow[b]{2}{*}{ SOURCE } & \multicolumn{4}{|c|}{ Dense Cloud Dust Features } & \multicolumn{6}{|c|}{ Diffuse Cloud Dust Features } \\
\hline & $\tau_{3.0}$ & $\tau_{4.27}$ & $\tau_{6.13}{ }^{b}$ & $\tau_{7.68}$ & $\tau_{3.28}$ & $\tau_{3.4}$ & $\tau_{6.2}{ }^{\mathrm{c}}$ & $\tau_{6.85}$ & $\tau_{7.25}$ & $\tau_{9.7}{ }^{\mathrm{d}}$ \\
\hline GCS $3 \ldots \ldots$ & $0.23 \pm 0.02$ & $0.48 \pm 0.02$ & $0.03 \pm 0.01$ & $<0.02$ & $0.026 \pm 0.005$ & $0.16 \pm 0.01$ & $0.10 \pm 0.02$ & $0.04 \pm 0.01$ & $<0.03$ & 2.38 \\
\hline GCS $4 \ldots \ldots$ & $0.16 \pm 0.01$ & $0.22 \pm 0.02$ & $0.02 \pm 0.01$ & $<0.03$ & $<\overline{0.05}$ & $0.15 \pm 0.01$ & $0.08 \pm 0.02$ & $<0.03$ & $<0.03$ & 2.24 \\
\hline
\end{tabular}

a Optical depth values for GCS 3 are from AOT6 data where available; $\tau_{7.68}$ for Sgr A* is from AOT6 data; all others are determined from AOT1 spectra.

${ }^{b}$ Optical depth is from the maximum allowable depth of the $\mathrm{H}_{2} \mathrm{O}: \mathrm{NH}_{3}: \mathrm{CO}_{2}$ mixture at $6.13 \mu \mathrm{m}$, which is constrained by the depth of the $3 \mu \mathrm{m}$ feature. See $\S 4.1$ for details.

c Optical depth is that of the residual feature after subtracting the ice component. See $\S \S 4.1$ and 5.2.1 for details.

${ }^{d}$ Silicate optical depths from Schutte et al. 1998 and Vriend (2000). 


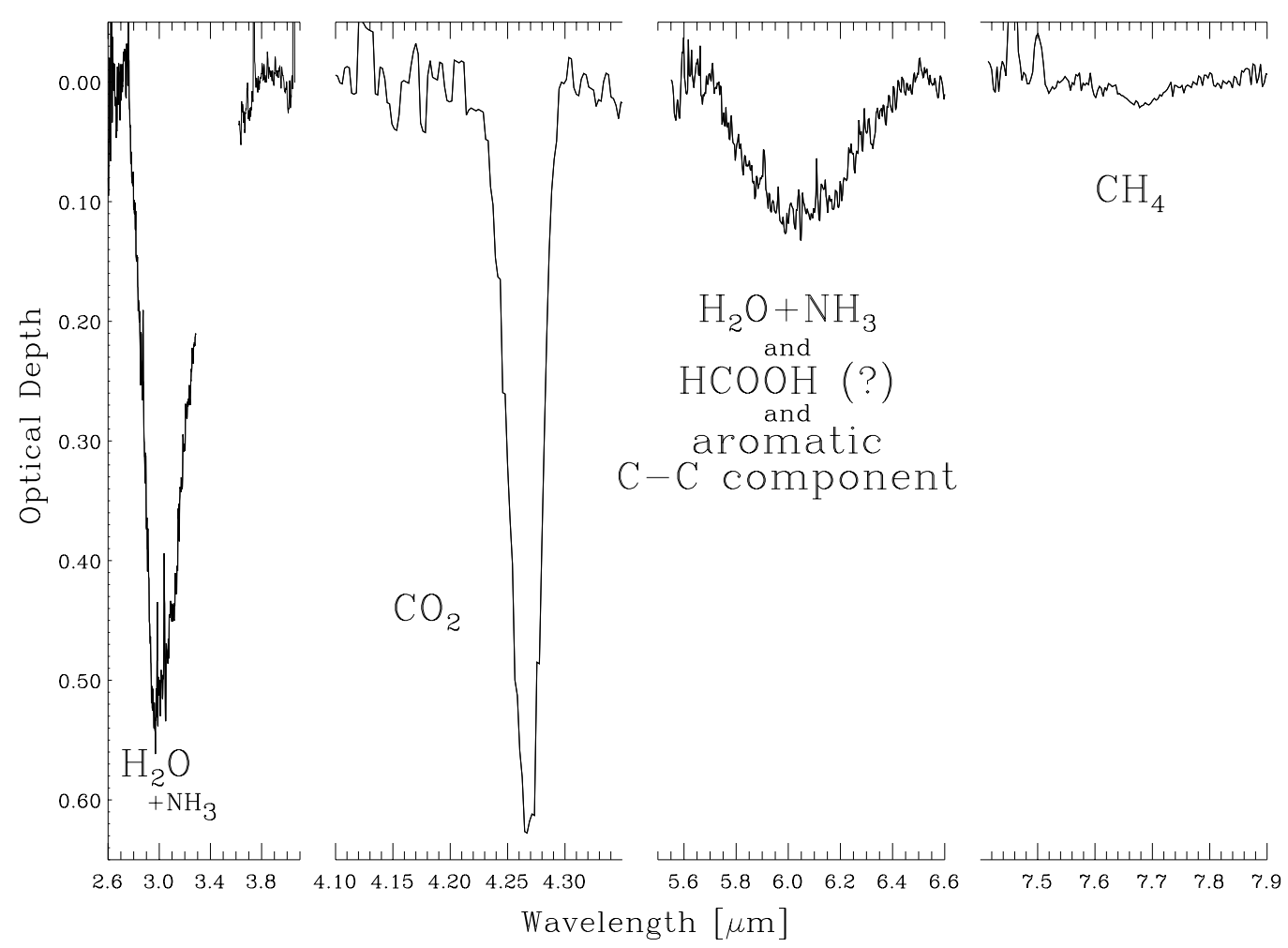

FIG. 2.- Molecular cloud dust absorption features identified in Sgr A*. To highlight the molecular cloud features, the 3.29-3.62 region has been removed from the "ice" profile. All data are AOT1, speed 4, except for the $7 \mu \mathrm{m}$ region, which is AOT6.

ent from the $\mathrm{H}_{2} \mathrm{O}$-ice absorption features observed toward molecular clouds elsewhere in the Galaxy (McFadzean et al. 1989; Butchart et al. 1986). Figure 3 compares the $3 \mu \mathrm{m}$ water-ice feature observed in the Taurus dark cloud in the line of sight toward the background K-giant Elias 16 (from Smith, Sellgren, \& Tokunaga 1989) with those of the GC sources, Sgr A*, GCS 3, and GCS 4. Clearly, the $3 \mu \mathrm{m}$ band in the Galactic center shows a blue absorption wing peaking at about $2.95 \mu \mathrm{m}$. (The aliphatic hydrocarbons absorption band at $3.4 \mu \mathrm{m}$ is discussed in $\S 5.1$ ). While initially this difference in profile was taken to imply that this band might be caused by water of hydration in silicates rather than $\mathrm{H}_{2} \mathrm{O}$ ice (Tielens et al. 1996; Wada et al. 1991; Tielens \& Allamandola 1987), the absence of this band in local sight lines through the diffuse ISM sheds some doubt on that interpretation (e.g., Whittet et al. 1997). The discovery of solid $\mathrm{CO}_{2}$ by $\mathrm{ISO}$ now firmly links the $3.0 \mu \mathrm{m}$ band in the GC to $\mathrm{H}_{2} \mathrm{O}$ ices (de Graauw et al. 1996b; Lutz et al. 1996; Whittet et al. 1997; Gerakines et al. 1999).

We consider the possibility that the $3.0 \mu \mathrm{m}$ band could be caused by diluted mixtures of $\mathrm{H}_{2} \mathrm{O}$ with other molecules, such as $\mathrm{CO}, \mathrm{O}_{2}$, and $\mathrm{NH}_{3}$, as suggested by Schutte \&
Greenberg (1989). Drawing on extensive laboratory studies by the Leiden (http://www.strw.leidenuniv.nl/ lab/; Gerakines et al. 1995, 1996) and NASA/Ames Astrophysics Laboratory groups, we attribute the difference in the $3.0 \mu \mathrm{m}$ profile between the GC and local molecular clouds to a higher abundance of other species - notably ammonia - in the $\mathrm{H}_{2} \mathrm{O}$ ice in the former relative to the latter regions. Possibly, this increased abundance of $\mathrm{NH}_{3}$ ice in the GC region relative to $\mathrm{H}_{2} \mathrm{O}$ ice reflects the increase in the $\mathrm{N} / \mathrm{O}$ elemental abundance ratio with decreasing galactocentric radius (Simpson et al. 1995; Rubin et al. 1988). A detailed discussion of the laboratory results for $\mathrm{H}_{2} \mathrm{O}: \mathrm{NH}_{3}$ mixtures will be discussed in a subsequent paper (J. Chiar \& M. Bernstein, 2000, in preparation). We describe the laboratory fits to the $3 \mu \mathrm{m}$ profile and determination of $\mathrm{H}_{2} \mathrm{O}$ ice column density along with the discussion of the $6 \mu \mathrm{m}$ ice profile below.

\subsubsection{The $6 \mu \mathrm{m}$ Feature}

The 5.5-6.5 $\mu \mathrm{m}$ spectral region contains the vibrational modes of several molecules of astrophysical interest: $\mathrm{O}-\mathrm{H}$ bend in $\mathrm{H}_{2} \mathrm{O}(6.0 \mu \mathrm{m}), \mathrm{C}=\mathrm{O}$ stretch in carbonyl groups

TABLE 3

ICE COLUMN DensitIES ${ }^{\mathrm{a}}$

\begin{tabular}{|c|c|c|c|c|c|c|c|}
\hline Source & $N\left(\mathrm{H}_{2} \mathrm{O}\right)$ & $N\left(\mathrm{CO}_{2}\right)^{\mathbf{b}}$ & $N(\mathrm{CO})$ & $N\left(\mathrm{CH}_{4}\right)$ & $N(\mathrm{HCOOH})$ & $N\left(\mathrm{H}_{2} \mathrm{CO}\right)$ & $N\left(\mathrm{CH}_{3} \mathrm{OH}\right)$ \\
\hline Sgr A*...... & $12.4 \pm 2.5$ & $1.7 \pm 0.2$ & $<1.5$ & $0.30 \pm 0.07$ & $0.8 \pm 0.2$ & $<0.3$ & $<0.5$ \\
\hline GCS $3 \ldots \ldots$ & $4.7 \pm 0.4$ & $1.1 \pm 0.1$ & $<0.9$ & $<0.3$ & $\ldots$ & $\ldots$ & $\ldots$ \\
\hline GCS $4 \ldots \ldots$ & $3.0 \pm 0.3$ & $0.7 \pm 0.2$ & $<0.9$ & $<0.5$ & $\ldots$ & $\ldots$ & $\ldots$ \\
\hline
\end{tabular}

${ }^{a}$ Column densities in units of $10^{17} \mathrm{~cm}^{-2}$.

${ }^{\mathrm{b}}$ Gerakines et al. 1999. 


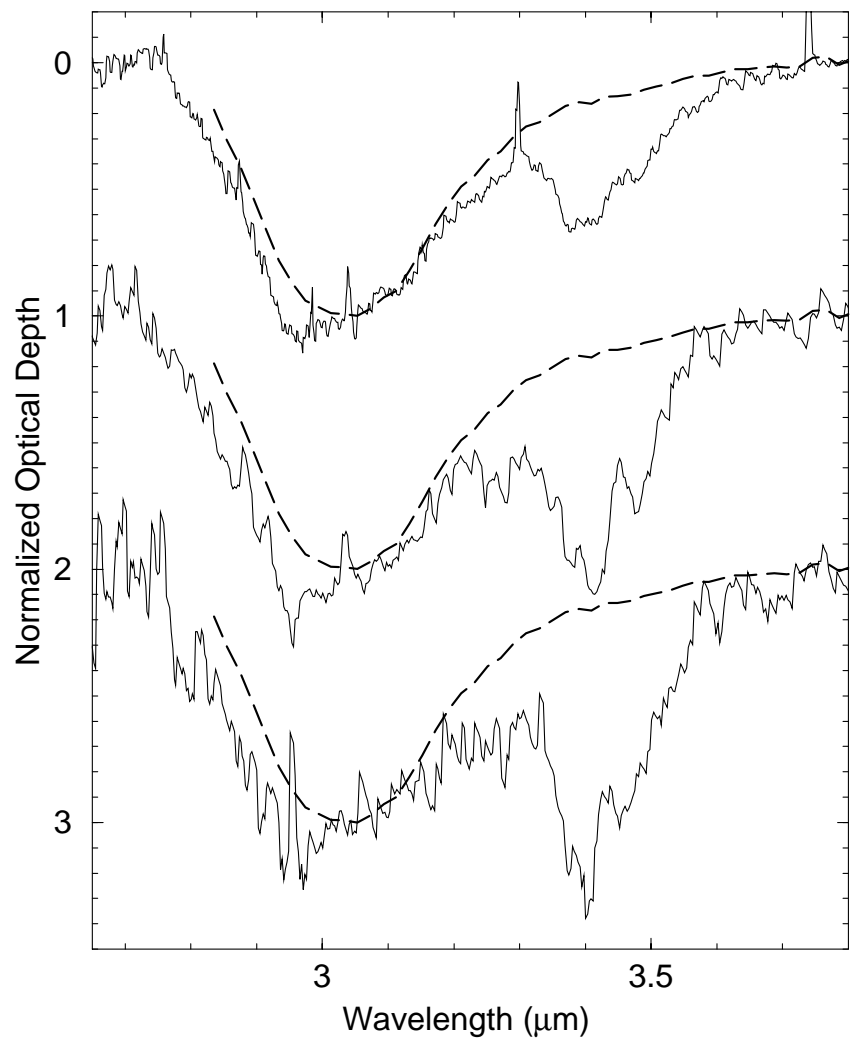

FIG. 3.-Optical depth profiles of the $3 \mu \mathrm{m}$ ice feature for Taurus dense cloud (Elias 16, dashed line; adapted from Smith et al. 1989) compared with the Galactic center sources Sgr A* (top), GCS 3 (middle), GCS 4 (bottom). All GC sources show excess blue absorption compared to the Taurus ice profile. Spectra have been normalized to have a depth of 1.0 at $3.05 \mu \mathrm{m}$.

(occurring in aldehydes, ketones, and carboxylic acids; $5.7-5.9 \mu \mathrm{m})$, and $\mathrm{C}-\mathrm{C}$ stretch in aromatic materials $(6.2$ $\mu \mathrm{m})$. The spectra of all GC sources show an absorption feature around $6.0 \mu \mathrm{m}$ (Fig. 4). Sources in or behind local molecular clouds also show a feature at $6.0 \mu \mathrm{m}$ (see also Fig. 6) caused mostly by the $\mathrm{O}-\mathrm{H}$ bending mode in $\mathrm{H}_{2} \mathrm{O}$ ice, although trace amounts of other molecules may contribute to the absorption (Keane et al. 2000; Schutte et al. 1996b; Tielens \& Allamandola 1987). In contrast, Galactic WolfRayet WC-type stars show an absorption feature at $6.2 \mu \mathrm{m}$ attributed to the $\mathrm{C}-\mathrm{C}$ stretch in aromatic materials (Schutte et al. 1998; see also Fig. 4 and $\S$ 5.2.1). Visual inspection of Figure 4 reveals that the $6 \mu \mathrm{m}$ profile of Sgr A* is strikingly different than those of GCS 3 and GCS 4; the latter two instead show a similarity to the $6.2 \mu \mathrm{m}$ band observed toward late-type dusty WC stars such as WR118 (cf. § 5.2 and Schutte et al. 1998). Given the greater depth of the $3.0 \mu \mathrm{m}$ ice band in Sgr A*, compared to GCS 3 and GCS 4 (Fig. 1), the $6.0 \mu \mathrm{m}$ band of this source is likely to contain a dominant ice component.

Determination of the $6 \mu \mathrm{m}$ profile for Sgr A* depends heavily on the choice of continuum, which leads to uncertainties in the red side of the profile and peak optical depth and minor changes in the peak position. We carried out first- and second-degree polynomial fits to the 5.5-8.5 $\mu \mathrm{m}$ spectrum of Sgr A* to quantify the differences in the profiles resulting from each. Figures 5 and 6 show two possible continuum choices and the resulting optical depth spectra, respectively. Peak positions of $\lambda \sim 6.05$ (second-degree polynomial fit) and $6.10 \mu \mathrm{m}$ (first-degree polynomial fit),

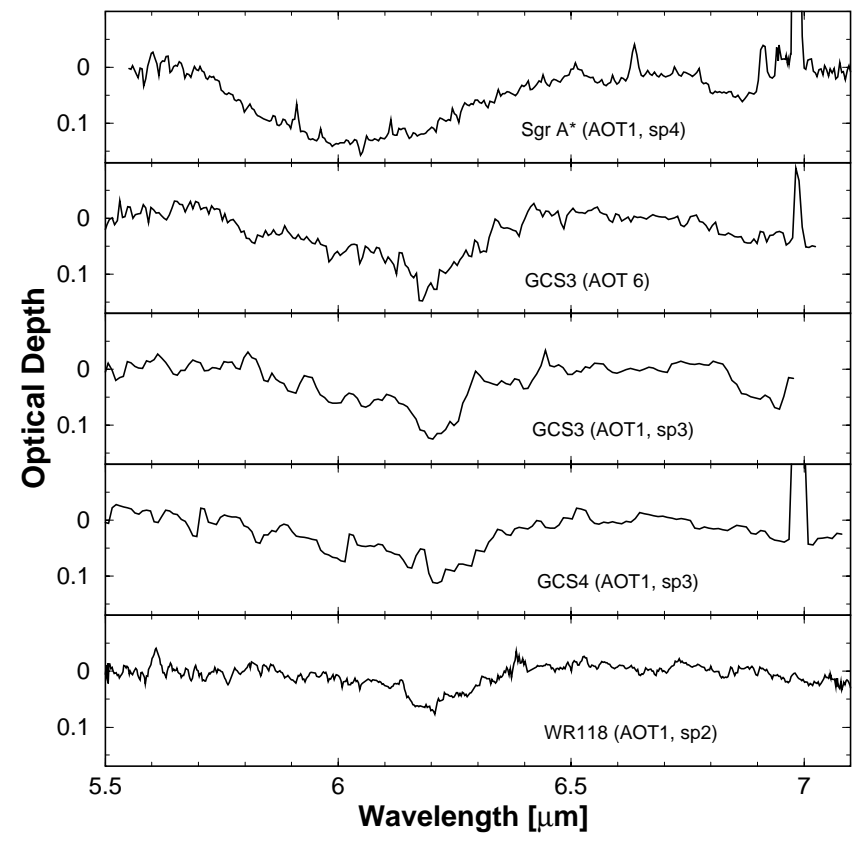

FIG. 4.-The $6.0 \mu \mathrm{m}$ region of Sgr A*, GCS 3, GCS 4, and WR 118. The spectrum of WR 118 is from Schutte et al. (1998). Narrow absorption lines (FWHM $\sim 0.01 \mu \mathrm{m}$ ) in the spectrum of GCS 3 (and Sgr A*) are caused by cold gas-phase $\mathrm{H}_{2} \mathrm{O}$ along the line of sight (Moneti \& Cernicharo 2000).

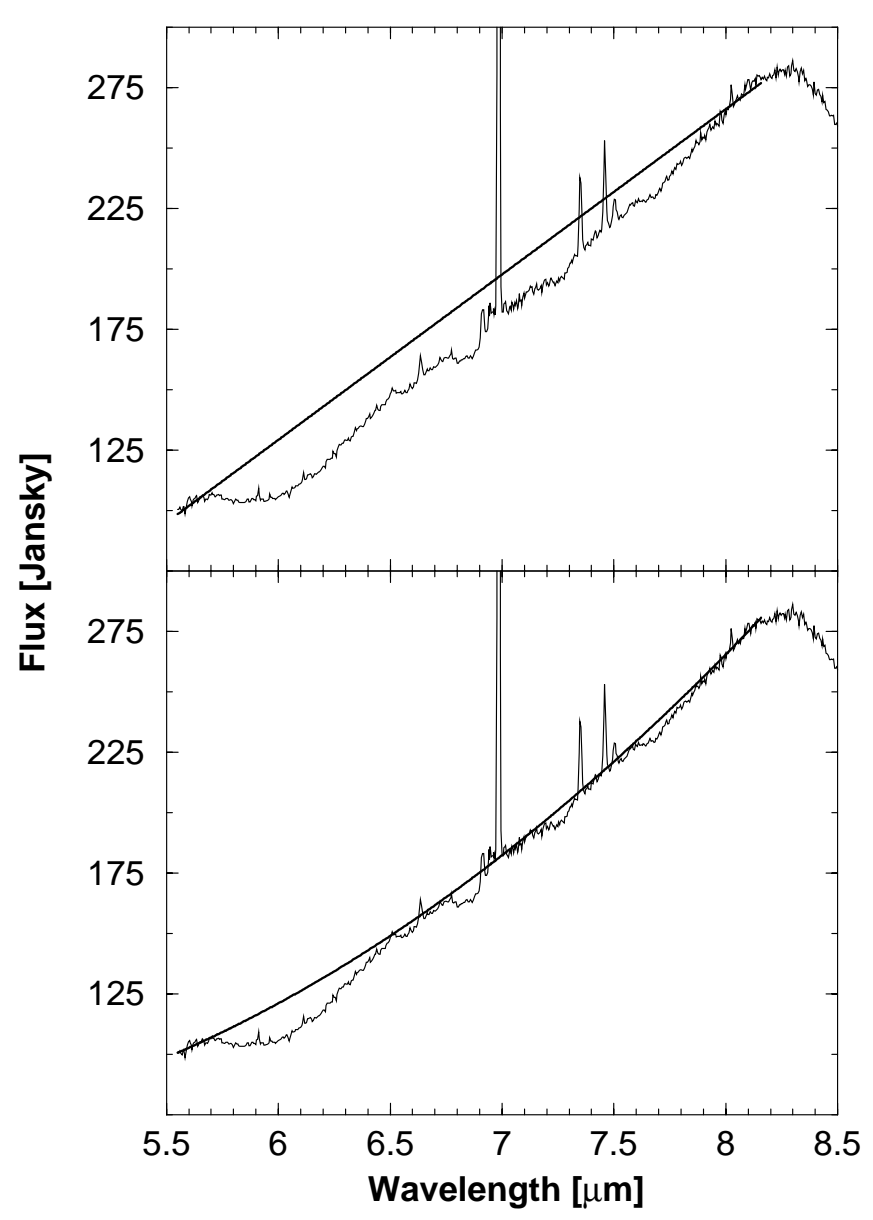

FIG. 5.-5.5-8.5 $\mu \mathrm{m}$ flux spectrum of Sgr A* showing second- (top) and first-degree (bottom) continuum fits. The resulting optical depth spectra are shown in Fig. 6. 


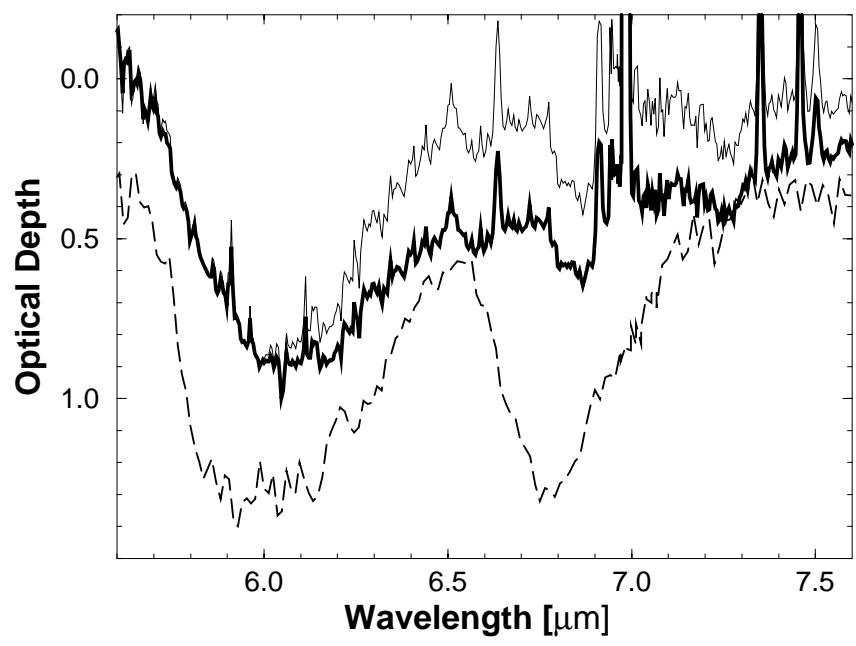

FIG. 6.-Comparison of the $6 \mu \mathrm{m}$ feature in Sgr A* using two different continuum fits as shown in Fig. 5. Optical depth spectra resulting from the second-degree polynomial fit (thin solid line) and first-degree polynomial fit (thick solid line) are shown. The optical depth spectrum of the protostellar object NGC 7538 IRS 9 (dashed line; Keane et al. 2000) is shown for comparison and is offset for clarity. All three spectra have been scaled to 1 at their peak optical depth.

and peak optical depths (at $6.05 \mu \mathrm{m}$ ) of 0.20 (first degree) and 0.14 (second degree) are obtained. We adopt the second-degree continuum fit, as this provides a good estimate of the continuum for the absorption features at 6.0 ,
6.85 , and $7.25 \mu \mathrm{m} .{ }^{9}$ For consistency, we also adopt seconddegree polynomial continuum fits for GCS 3 and GCS 4; the optical depth spectra are shown in Figure 4.

Rather than identifying the carrier of the $6 \mu \mathrm{m}$ feature in the GC sources in isolation, we use the $3 \mu \mathrm{m}$ profile as a constraint for the nature and abundances of the carriers of the corresponding $6 \mu \mathrm{m}$ feature. Most of the $6 \mu \mathrm{m}$ absorption in protostellar objects is explained by simple amorphous $\mathrm{H}_{2} \mathrm{O}$ ice mixtures, so we first attempt to match the GC 3 and $6 \mu$ m profiles with only pure $\mathrm{H}_{2} \mathrm{O}$ ice (using laboratory data from Leiden Observatory's database at http:// www.strw.leidenuniv.nl/ lab/). This procedure shows that fitting the $6 \mu \mathrm{m}$ band with pure $\mathrm{H}_{2} \mathrm{O}$ ice causes the optical depth of the $3 \mu \mathrm{m}$ band to be overestimated by a factor of 2-4.5 for all three objects. This is demonstrated in Figure 7 (bottom panels) for Sgr A* and in Figure 8 for GCS 3 (top panels) and GCS 4 (bottom panels). The mixture used, amorphous $\mathrm{H}_{2} \mathrm{O}$ at $30 \mathrm{~K}$, is a realistic analog for ices in these sight lines and provides the best fit to the Sgr A* $6 \mu \mathrm{m}$ profile when the $3 \mu \mathrm{m}$ spectrum is ignored. Such a discrepancy between the 3 and $6 \mu \mathrm{m} \mathrm{H}_{2} \mathrm{O}$ ice optical depth has been noted for some massive young stellar objects (Gibb et al. 2000; Keane et al. 2000). For these sources, scattering at near-IR wavelengths in the disk-like environment of the protostar may reduce the apparent optical depth at $3.0 \mu \mathrm{m}$

${ }^{9}$ A local continuum in the 7.3-8.0 $\mu$ m region is used for the $\mathrm{CH}_{4}$ feature at $7.68 \mu \mathrm{m}$. See $\S 4.3$ for further discussion.

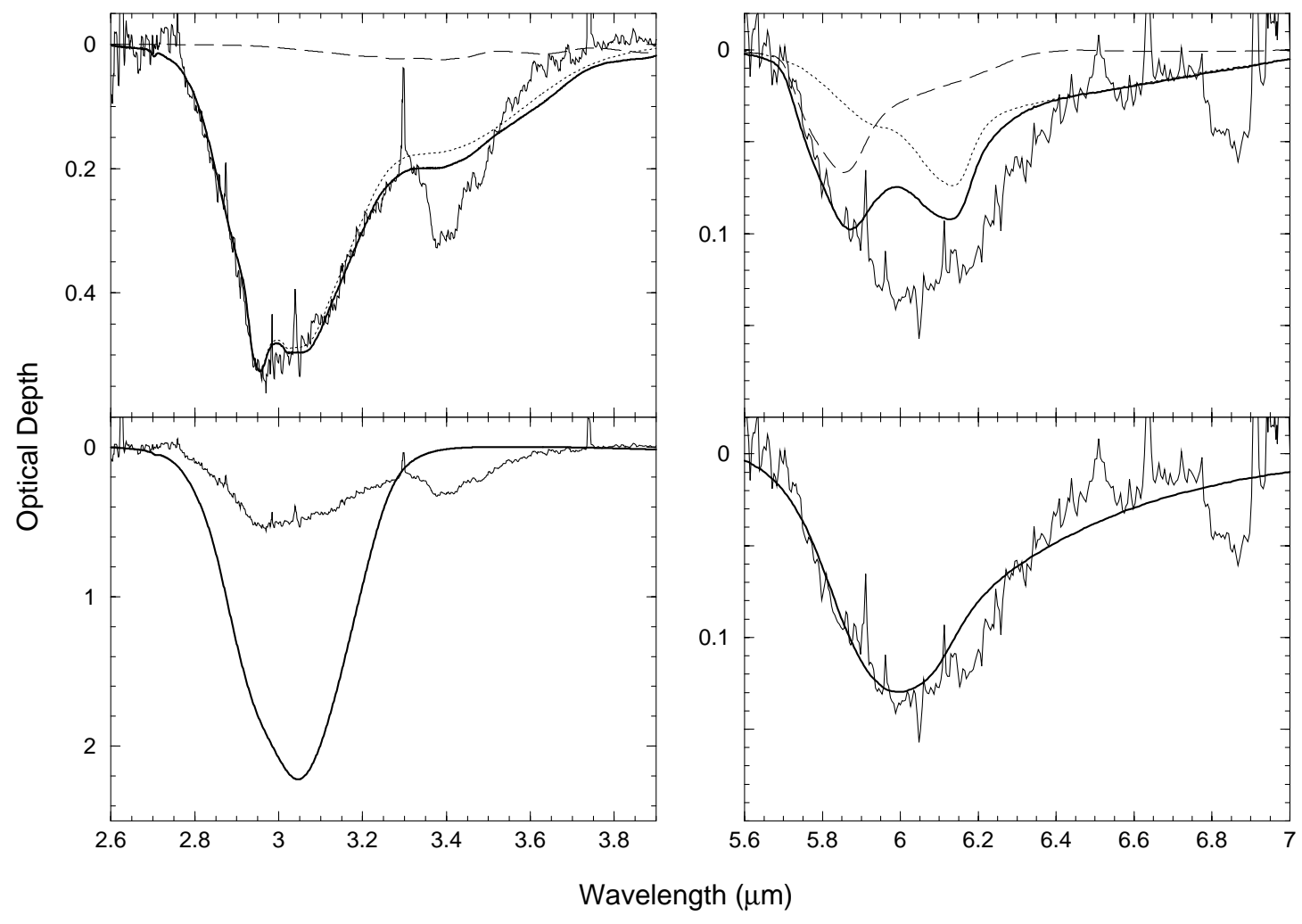

Fig. 7.-2.6-3.9 $\mu \mathrm{m}$ (left panels) and 5.6-7 $\mu \mathrm{m}$ (right panels) optical depth spectra for Sgr A*. The top panels show the result of the fitting procedure when both profiles are used to constrain the fit. The "best" fit is obtained with $\mathrm{H}_{2} \mathrm{O}: \mathrm{NH}_{3}: \mathrm{CO}_{2}(3: 1: 0.03,15 \mathrm{~K})$ (dotted line) and $\mathrm{HCOOH}$ at $10 \mathrm{~K}($ dashed line). The sum of the two laboratory mixtures is shown by the smooth solid line. The bottom panels show the result of the fitting procedure when only the $6 \mu \mathrm{m}$ profile is considered. In this case, although the $6 \mu \mathrm{m}$ band is reasonably matched with pure $\mathrm{H}_{2} \mathrm{O}$ at $30 \mathrm{~K}$ (smooth solid line), the depth of the $3 \mu \mathrm{m}$ profile is overestimated by the laboratory spectrum (bottom, left panel). 

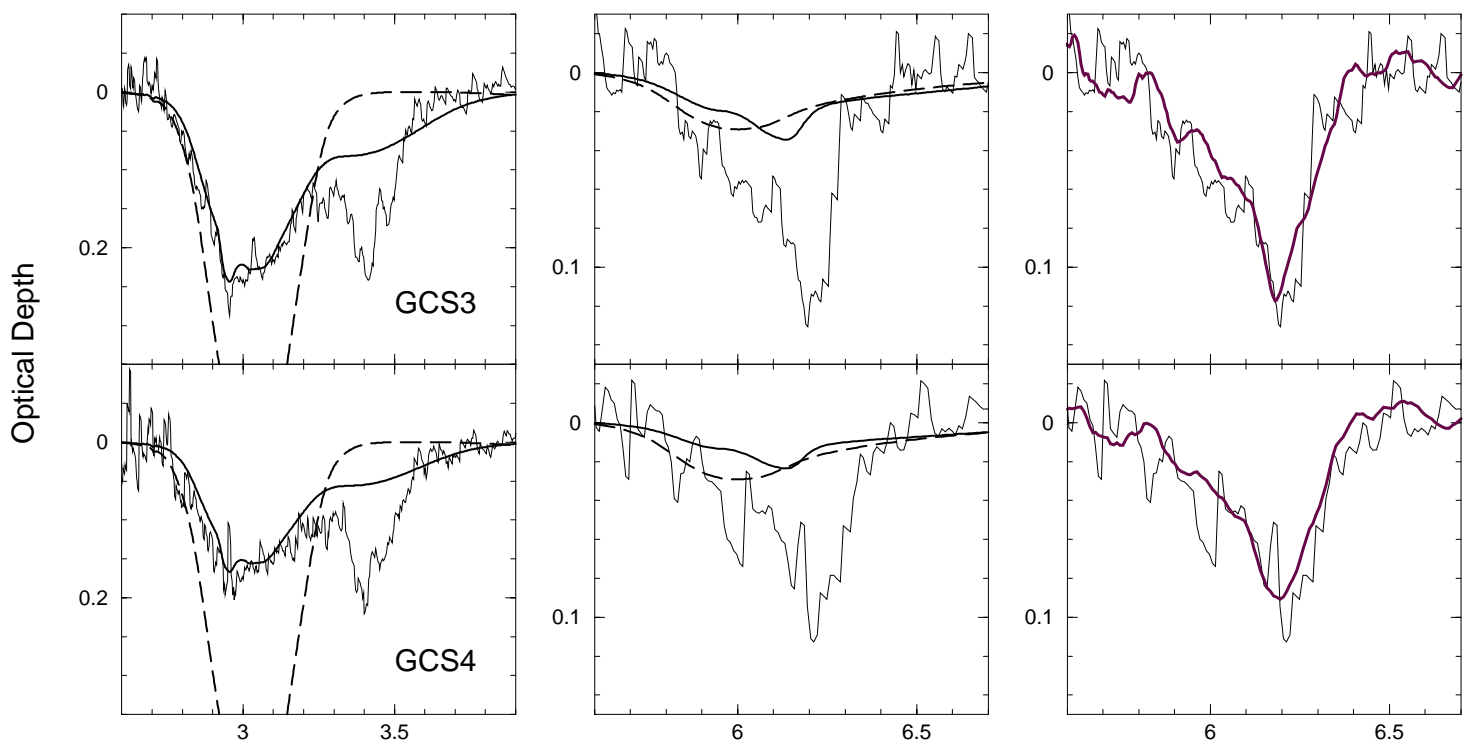

Wavelength $[\mu \mathrm{m}]$

FIG. 8.-2.6-3.9 $\mu \mathrm{m}$ (left panels) and 5.6-6.7 $\mu \mathrm{m}$ (center and right panels) spectral region of GCS 3 (top panels) and GCS 4 (bottom panels). Comparison of the laboratory mixtures $\mathrm{H}_{2} \mathrm{O}: \mathrm{NH}_{3}: \mathrm{CO}_{2}\left(3: 1: 0.03,15 \mathrm{~K}\right.$ ) (smooth solid line) and pure $\mathrm{H}_{2} \mathrm{O}$ at $30 \mathrm{~K}$ (dashed line) are shown for the 3 and $6 \mu \mathrm{m}$ spectral regions in the left and center panels, respectively. Similar to Sgr A*, the $\mathrm{H}_{2} \mathrm{O}: \mathrm{NH}_{3}: \mathrm{CO}_{2}$ mixture represents these profiles better than pure $\mathrm{H}_{2} \mathrm{O}$ ice. The right panels show the result of summing the $\mathrm{H}_{2} \mathrm{O}: \mathrm{NH}_{3}: \mathrm{CO}_{2}$ mixture with a WR 118 -like $6.2 \mu$ m component (heavy solid line).

(Pendleton, Tielens, \& Werner 1990). For the Galactic center sources, ice absorption is not local to the sources but rather occurs along the line of sight and, hence, this effect should not play a role. Thus, we conclude that, despite the good fit, the $6.0 \mu \mathrm{m}$ band cannot be due to $\mathrm{H}_{2} \mathrm{O}$ ice alone.

The $3 \mu \mathrm{m}$ band toward Sgr $\mathrm{A}^{*}$ shows an excess blue absorption wing relative to other observed interstellar ice bands (cf. Fig. 3). This is likely because of the presence of $\mathrm{NH}_{3}$ in the ice (e.g., Schutte \& Greenberg 1989). There is evidence that the $6 \mu \mathrm{m}$ profile in protostellar objects is also due to a mixture of other molecules with $\mathrm{H}_{2} \mathrm{O}$ (Schutte et al. 1996b; Keane et al. 2000; Gibb et al. 2000). Thus, we favor a multicomponent "fit" to the 3 and $6 \mu \mathrm{m}$ ice features and have used the extensive databases at Leiden Observatory and NASA-Ames Astrophysical Laboratories to determine the carriers (in addition to $\mathrm{H}_{2} \mathrm{O}$ ice) responsible for the two features. These databases contain mixtures of $\mathrm{H}_{2} \mathrm{O}$ ice with $\mathrm{CH}_{3} \mathrm{OH}, \mathrm{CH}_{4}, \mathrm{O}_{2}, \mathrm{H}_{2} \mathrm{CO}, \mathrm{HCOOH}, \mathrm{CO}$, and $\mathrm{CO}_{2}$ as minor components, as well as pure ices of these molecules, at temperatures ranging from 10 to $245 \mathrm{~K}$ (http:// www.strw.leidenuniv.nl/ lab/; J. Chiar \& M. Bernstein, 2000 , in preparation). A formal chi-squared fitting routine is used to fit the profiles in the Sgr A* spectrum. The "best" fit, shown in Figure 7 (top panels), consists of $\mathrm{H}_{2} \mathrm{O}: \mathrm{NH}_{3}: \mathrm{CO}_{2}(100: 30: 6,15 \mathrm{~K})$ and $\mathrm{HCOOH}$ at $10 \mathrm{~K}$. Summing these mixtures provides an impressive fit to the 3 $\mu \mathrm{m}$ feature, including the so-called long-wavelength wing (3.4 $\mu \mathrm{m}$ absorption is caused by diffuse ISM dust; this region is excluded from the fit), and can account for much of the absorption at $6 \mu \mathrm{m}$. Formaldehyde $\left(\mathrm{H}_{2} \mathrm{CO}\right)$ has been discussed as a candidate for the red side of the $6 \mu \mathrm{m}$ feature in protostellar objects (Schutte et al. 1996a; Keane et al. 2000); the Sgr A* profile (which is tightly constrained on the red side, regardless of continuum choice) limits the amount of $\mathrm{H}_{2} \mathrm{CO}$ relative to $\mathrm{H}_{2} \mathrm{O}$ ice to $\sim 2 \%$.
Residual absorption resulting from subtracting the "fit" from the astronomical data is shown in Figure 9 (top panel). Much of this remaining absorption is accounted for by a component at $6.2 \mu \mathrm{m}$, similar to the WR $1186.2 \mu \mathrm{m}$ profile (Figs. 4, 9). See $\S 5.2 .1$ for a detailed discussion of this component. Only a small portion of the $6 \mu \mathrm{m}$ profile remains unaccounted for in the Sgr A* spectrum (Fig. 9, bottom panel). Additional organic acid mixtures, such as $\mathrm{HCOOH}$ at a range of temperatures and host matrices, are needed to resolve this issue.

The weakness of the $6 \mu \mathrm{m}$ ice components in GCS 3 and GCS 4 make a chi-squared analysis of the 3 and $6 \mu \mathrm{m}$ bands unwarranted (see the middle panels in Fig. 8); however, their $3 \mu \mathrm{m}$ profiles and the relative depths of their 3 and $6 \mu \mathrm{m}$ bands can be used to help constrain the nature of the ices. Pure $\mathrm{H}_{2} \mathrm{O}$ does not provide a satisfactory match to the 3 and $6 \mu \mathrm{m}$ profiles, as discussed above (Fig. 8). The $3 \mu \mathrm{m}$ profiles of these sources are well matched by the same $\mathrm{H}_{2} \mathrm{O}: \mathrm{NH}_{3}: \mathrm{CO}_{2}$ mixture used to fit the Sgr A* profiles, which is suggestive of a consistent $\mathrm{NH}_{3} / \mathrm{H}_{2} \mathrm{O}$ ratio for dense clouds toward the Galactic center. A component like the WR $1186.2 \mu \mathrm{m}$ feature accounts for the remainder of the 6 $\mu \mathrm{m}$ profile, as for Sgr A*. Figure 8 shows that all of the $6 \mu \mathrm{m}$ absorption in the Quintuplet spectra is accounted for by the sum of the ice mixture $\left(\mathrm{H}_{2} \mathrm{O}: \mathrm{NH}_{3}: \mathrm{CO}_{2}, 15 \mathrm{~K}\right)$ and a $6.2 \mu \mathrm{m}$ "diffuse ISM" component (represented by the WR 118 spectrum). Detailed discussion of the $6.2 \mu \mathrm{m}$ feature is deferred to $\S$ 5.2.1.

To summarize, using the available databases of interstellar ice analogs, the 3 and $6 \mu \mathrm{m}$ profiles observed toward Sgr A*, GCS 3, and GCS 4 are best represented by the ice mixture, $\mathrm{H}_{2} \mathrm{O}: \mathrm{NH}_{3}: \mathrm{CO}_{2}(100: 30: 6,15 \mathrm{~K})$, with a small amount of $\mathrm{HCOOH}$ at $10 \mathrm{~K}$ (for $\mathrm{Sgr} \mathrm{A}^{*}$ ), in addition to a diffuse ISM component at $6.2 \mu \mathrm{m}$. Pure $\mathrm{H}_{2} \mathrm{O}$ cannot account for the shape of the $3 \mu \mathrm{m}$ profile or the relative depths of the 3 and $6 \mu \mathrm{m}$ features. Water-ice column den- 


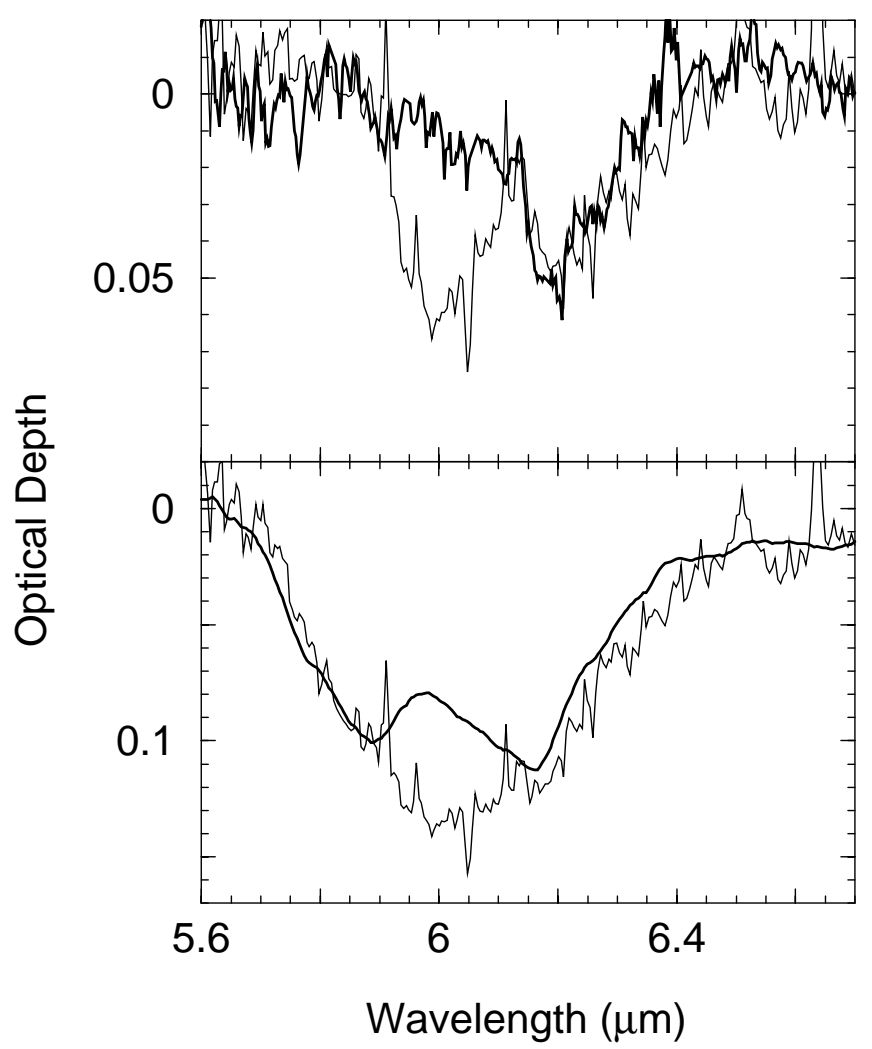

FIG. 9.-(Top panel) $6 \mu \mathrm{m}$ residual for Sgr A* obtained after subtracting the best-fitting laboratory mixtures shown in Fig. 7. The heavy line represents the WR $1186.2 \mu \mathrm{m}$ optical depth spectrum, divided by a factor of 1.25. The bottom panel shows the "fit" resulting from the summation of the best-fitting laboratory ices and the scaled WR $1186.2 \mu$ m feature.

sities (Table 3) are determined by the fit to the $3 \mu \mathrm{m}$ profile (Fig. 7 [top left, dotted line], Fig. 8 [left panels, solid line]), using $N=\int \tau_{v} d v / A$, and $A=2 \times 10^{-16} \mathrm{~cm}$ molecule ${ }^{-1}$ (Hagen, Tielens, \& Greenberg 1981). Column density for $\mathrm{HCOOH}$ is determined using the $6 \mu \mathrm{m}$ fit (Fig. 7 [top right, dashed line $]$ ), and $A=6.7 \times 10^{-17} \mathrm{~cm}$ molecule $^{-1}$ (Maréchal 1987).

\section{2. $\mathrm{CO}_{2}$ and $\mathrm{CH}_{3} \mathrm{OH}$ Ice and $\mathrm{CO}$}

Solid $\mathrm{CO}_{2}$ absorption features at $4.27 \mu \mathrm{m}$ (stretching mode) and $15.2 \mu \mathrm{m}$ (bending mode) have been discussed extensively by Gerakines et al. (1999). Fits to the $\mathrm{CO}_{2}$ profiles showed that toward the sources discussed in this paper, $\mathrm{CO}_{2}$ ice is embedded in a mainly polar matrix at temperatures less than $50 \mathrm{~K}$.

We searched for solid $\mathrm{CH}_{3} \mathrm{OH}$ absorption features at $3.54 \mu \mathrm{m}(\mathrm{C}-\mathrm{H}$ stretch), $3.9 \mu \mathrm{m}$ (combination mode), and $6.85 \mu \mathrm{m}(\mathrm{C}-\mathrm{H}$ deformation mode $)$ in the Sgr A* spectrum. The $\mathrm{C}-\mathrm{H}$ stretch mode occurs in the red wing of the $3.4 \mu \mathrm{m}$ aliphatic hydrocarbon feature. In order to obtain a limit on the depth of a possible $\mathrm{CH}_{3} \mathrm{OH}$ feature at $3.54 \mu \mathrm{m}$, a local second-degree polynomial was fitted between 3.4 and 3.7 $\mu \mathrm{m}$. A second-degree polynomial continuum was fitted in the 3.6-4.1 $\mu \mathrm{m}$ region to estimate the $3.9 \mu \mathrm{m}$ feature depth. Although we detect a feature centered at $6.85 \mu \mathrm{m}$ toward $\mathrm{Sgr} \mathrm{A}$, its width is too narrow to be caused by $\mathrm{CH}_{3} \mathrm{OH}$ (see $\S 5.1$ ), thus the continuum on either side of the observed 6.85 $\mu \mathrm{m}$ feature is used to deduce a limit for the $\mathrm{CH}_{3} \mathrm{OH}$ feature. Limits on the optical depths are $\tau_{3.54}<0.01, \tau_{3.9}<0.001$, and $\tau_{6.85}<0.005$. Using the integrated absorbance values for pure $\mathrm{CH}_{3} \mathrm{OH}\left(A=5.3 \times 10^{-18}, 2.8 \times 10^{-18}\right.$, $1.2 \times 10^{-17} \mathrm{~cm}^{2}$ molecule ${ }^{-1}$, for the $3.54,3.9$, and $6.85 \mu \mathrm{m}$ features, respectively; Hudgins et al. 1993), the average calculated $\mathrm{CH}_{3} \mathrm{OH}$ column density is less than $0.5 \times 10^{17}$ $\mathrm{cm}^{-2}$, resulting in an abundance of less than 4\% relative to $\mathrm{H}_{2} \mathrm{O}$ ice. This is similar to the value $N\left(\mathrm{CH}_{3} \mathrm{OH}\right) / N\left(\mathrm{H}_{2} \mathrm{O}\right)<5 \%$ found for the quiescent Taurus dark cloud (Chiar, Adamson, \& Whittet 1996) and significantly less than the abundance found for some high-mass protostars toward which $N\left(\mathrm{CH}_{3} \mathrm{OH}\right) / N\left(\mathrm{H}_{2} \mathrm{O}\right)$ is as high as 30\% (Allamandola et al. 1992; Skinner et al. 1992; Dartois et al. 1999).

Carbon monoxide is present primarily in the gas phase in these lines of sight. Limiting values for the solid CO column densities derived in this work are listed in Table 3. These values agree with those independently analyzed by Gerakines et al. (1999).

\section{3. $\mathrm{CH}_{4}$ Ice}

The deformation mode of solid methane $\left(\mathrm{CH}_{4}\right)$ occurs near $7.68 \mu \mathrm{m}$ and has been detected in three protostellar sources to date (d'Hendecourt et al. 1996; Boogert et al. 1996, 1998). We detect this absorption feature in the Sgr A* and Sgr A W-IRS 3 spectra with $\tau_{7.68} \simeq 0.02$ (Fig. 10). Fits to the higher quality Sgr A W-IRS 3 AOT6 spectrum were carried out using the database at Leiden Observatory (Boogert et al. 1997). This spectrum is best-fitted by $\mathrm{CH}_{4}$ embedded in a polar matrix containing molecules such as $\mathrm{H}_{2} \mathrm{O}$ and/or $\mathrm{CH}_{3} \mathrm{OH}$ at temperatures ranging from 10 to 80 $\mathrm{K}$. Nonpolar mixtures do not provide a good fit as they peak at shorter wavelengths and have more narrow profiles than the observed feature. Our result is consistent with recent studies of solid $\mathrm{CH}_{4}$ toward protostellar objects that have shown that $\mathrm{CH}_{4}$ embedded in a polar matrix provides

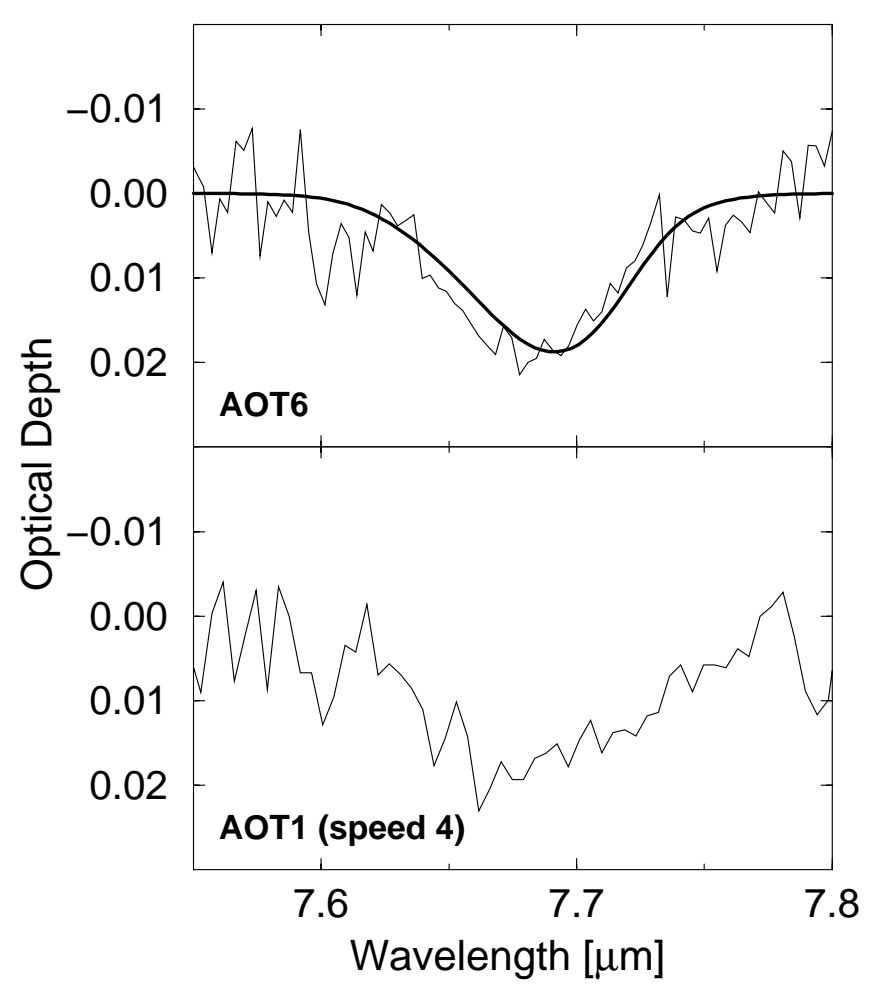

FIG. 10.-7.55-7.8 $\mu \mathrm{m}$ optical depth spectrum of Sgr A* shown with best-fitting laboratory mixture $\mathrm{H}_{2} \mathrm{O}: \mathrm{CH}_{4}(3: 1,10 \mathrm{~K})$ (smooth solid line). 
the best fit to the observed profile (Boogert et al. 1996; d'Hendecourt et al. 1996). We adopt the matrixindependent integrated absorption strength, $A=7.3 \times 10^{-18} \mathrm{~cm}$ molecule ${ }^{-1}$ to compute the column density of $\mathrm{CH}_{4}$ toward Sgr A*: $N\left(\mathrm{CH}_{4}\right)=3 \pm 1 \times 10^{16}$ $\mathrm{cm}^{-2}$, resulting in $N\left(\mathrm{CH}_{4}\right) / N\left(\mathrm{H}_{2} \mathrm{O}\right)=0.02 \pm 0.01$, within the range, $0.4 \%-4 \%$, found for protostellar objects (Boogert et al. 1996; d'Hendecourt et al. 1996).

The peak optical depth of the $\mathrm{C}-\mathrm{H}$ stretching mode of solid $\mathrm{CH}_{4}$, at $3.32 \mu \mathrm{m}$, is expected to be the same as that of the deformation mode (Hudgins et al. 1993; Boogert et al. 1997). Since this feature falls in the blue wing of the $3.4 \mu \mathrm{m}$ aliphatic hydrocarbon feature (§ 5.1), its presence cannot be confirmed.

Assuming a similar $N\left(\mathrm{CH}_{4}\right) / N\left(\mathrm{H}_{2} \mathrm{O}\right)$ ratio for GCS 3 and GCS 4 as for Sgr A*, a feature with optical depth $\tau_{7.68}<$ 0.01 is expected for the $\mathrm{C}-\mathrm{H}$ deformation mode. The current data do not have a sufficient signal-to-noise ratio in this wavelength region to detect such a weak feature; limiting values for $\tau_{7.68}$ and $N\left(\mathrm{CH}_{4}\right)$ are given in Tables 2 and 3 , respectively.

\section{THE DIFFUSE ISM FEATURES}

Aliphatic hydrocarbons are known to be a widespread component of the diffuse ISM, as is shown by the ubiquitous presence of the $3.4 \mu \mathrm{m} \mathrm{C}-\mathrm{H}$ stretch absorption feature in all studied sight lines. ISO-SWS data have now given us irrefutable evidence for the corresponding deformation modes at longer wavelengths. Contrarily, aromatic hydrocarbons, which have $\mathrm{C}-\mathrm{H}$ and $\mathrm{C}-\mathrm{C}$ stretch modes at $\sim 3.3$ and $6.2 \mu \mathrm{m}$, respectively, are not yet well established as a universal component of the diffuse ISM. We discuss these hydrocarbon features toward the GC in the following sections. Aliphatic hydrocarbon features detected toward
Sgr $A^{*}$ are shown in Figure 11; Figure 12 displays both aliphatic and aromatic hydrocarbon absorption features observed toward GCS 3.

\subsection{The 3.4, 6.85, and 7.25 $\mu \mathrm{m}$ Aliphatic Hydrocarbon Features}

Saturated aliphatic hydrocarbons exhibit $\mathrm{CH}$ stretching modes at $\sim 3.4 \mu$ m (methyl and methylene groups) and $\mathrm{CH}$ deformation modes at $\sim 6.9 \mu \mathrm{m}$ (methyl and methylene groups) and $\sim 7.3 \mu \mathrm{m}$ (methyl group). The profile of the 3.4 $\mu \mathrm{m}$ absorption features toward Sgr A* (Fig. 11), GCS 3 (Fig. 12 ), and GCS 4 is consistent with previous ground-based observations: substructure in the feature is identified with the $\mathrm{CH}$ stretch in methyl $\left(-\mathrm{CH}_{3}\right)$ and methylene $\left(-\mathrm{CH}_{2}-\right)$ groups, with an average ratio of $-\mathrm{CH}_{2}-/-\mathrm{CH}_{3} \simeq 2-2.5$, in saturated aliphatic hydrocarbons (Pendleton et al. 1994; Sandford et al. 1991). Subfeatures caused by the symmetric and asymmetric vibrational stretching modes of the $\mathrm{CH}$ groups are indicated in the 3.2-3.9 $\mu \mathrm{m}$ optical depth spectrum for GCS 3 (Fig. 12).

The Sgr A* spectrum shows clear absorption features at 6.85 and $7.25 \mu \mathrm{m}$ (Fig. 11) caused by the asymmetric and symmetric $\mathrm{CH}$ deformation modes, respectively. The 6.85 $\mu \mathrm{m}$ band is also weakly present in the spectrum of GCS 3 , and may be present in the spectrum of GCS 4, consistent with the relatively weaker $3.4 \mu \mathrm{m}$ absorption features (Table 2, Fig. 4). We do not attempt to quantify the central wavelengths of the weak $6.85 \mu \mathrm{m}$ features in the GCS 3 and GCS 4 spectra; within reasonable uncertainties they are similar to that in Sgr A*. The signal-to-noise ratio of the GCS 3 and GCS 4 spectra in the $7 \mu \mathrm{m}$ region is not sufficient to detect the $7.25 \mu \mathrm{m}$ band; limits are given in Table 2. Using the Sgr A* spectrum, and assuming $-\mathrm{CH}_{2}-/-\mathrm{CH}_{3}=2.5$,

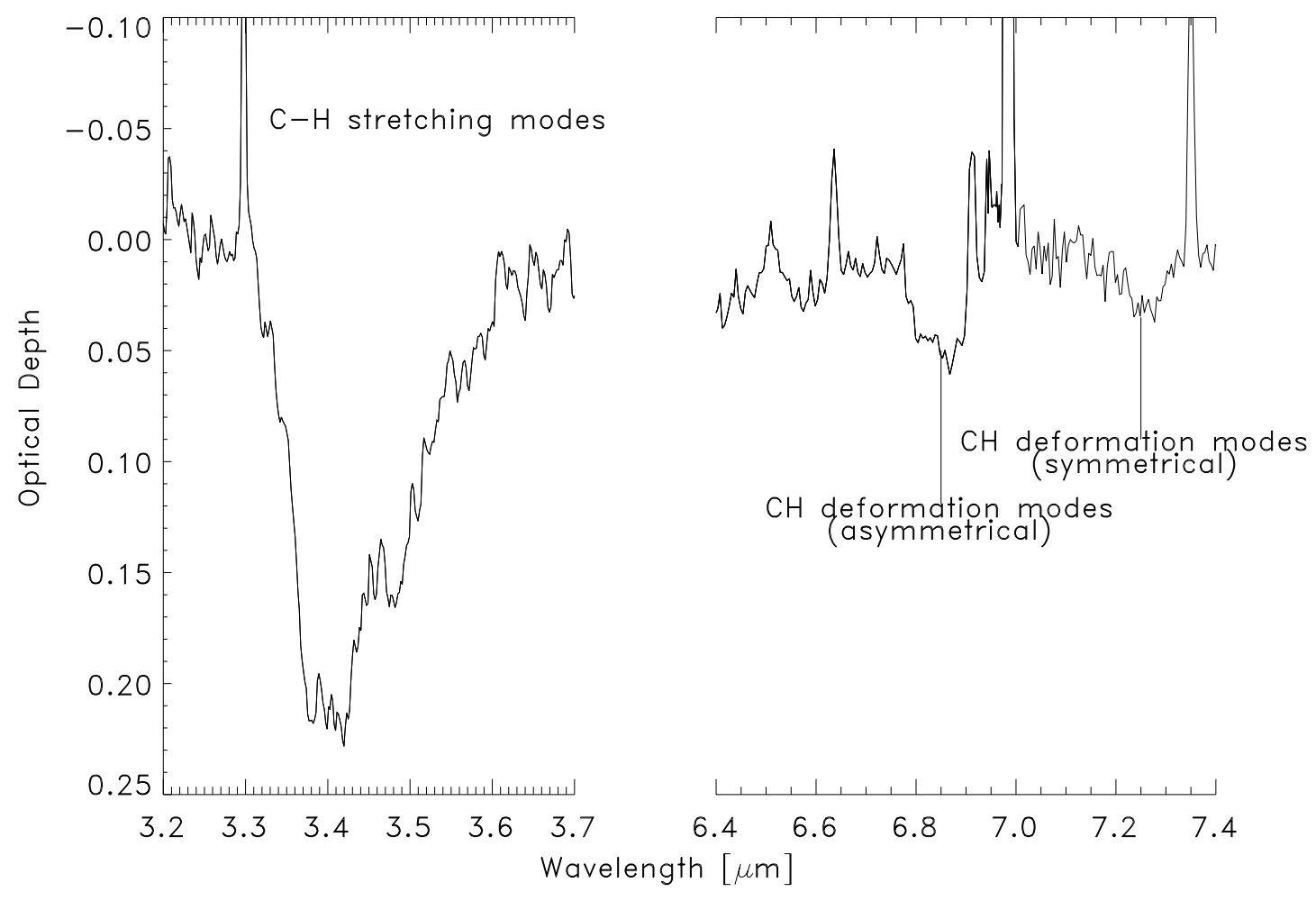

FIG. 11.-Aliphatic hydrocarbon absorption features identified in Sgr A* (AOT1 data). Shown are the well-known $3.4 \mu \mathrm{m}$ C-H stretch absorption feature and its corresponding deformation modes at 6.85 and $7.25 \mu \mathrm{m}$. 


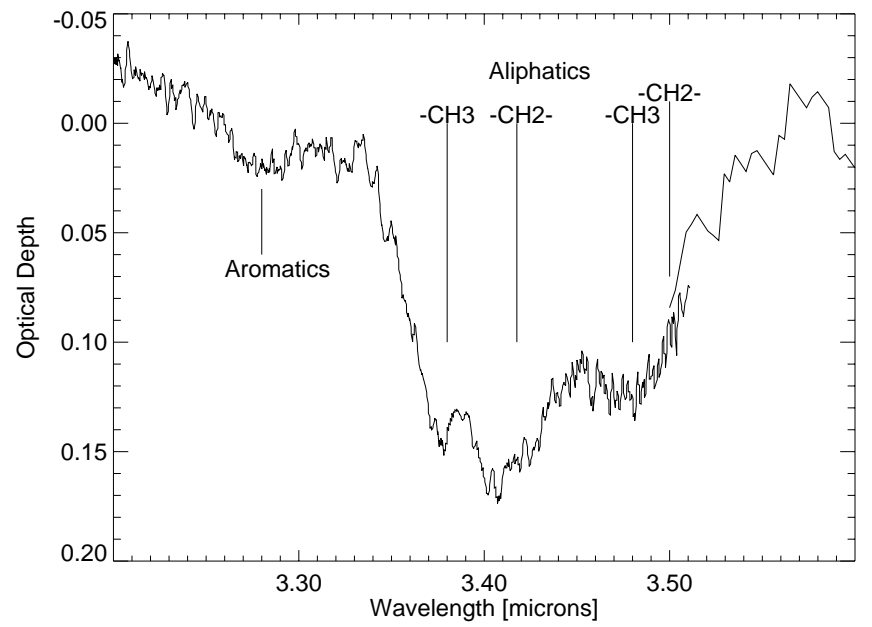

FIG. 12.- Hydrocarbon features toward GCS 3, combining AOT6 (3.2$3.5 \mu \mathrm{m})$ and AOT1 $(3.5-3.6 \mu \mathrm{m})$ data. The optical depth spectrum was created by fitting a local second-degree polynomial continuum to the combined spectra in 3.1-3.7 $\mu \mathrm{m}$ region. Aliphatic hydrocarbon subfeatures at $\sim 3.38 \mu \mathrm{m}\left(-\mathrm{CH}_{3}\right.$ asymmetric stretch), $3.42 \mu \mathrm{m}\left(-\mathrm{CH}_{2}-\right.$ asymmetric stretch), $3.48 \mu \mathrm{m}\left(-\mathrm{CH}_{3}\right.$ symmetric stretch), and $3.50 \mu \mathrm{m}\left(-\mathrm{CH}_{2}-\right.$ symmetric stretch) are indicated. The latter two subfeatures are blended. Also present is an aromatic hydrocarbon feature at $3.28 \mu \mathrm{m}$.

we calculated the expected ratio of integrated intensities for the 3.4, 6.85, and $7.25 \mu \mathrm{m}$ bands for branched and normal saturated aliphatic hydrocarbons from values by Wexler (1967) (Table 4). The 6.85 and $7.25 \mu \mathrm{m}$ feature profiles are estimated using Lorentzian curves with FWHM $=0.12 \mu \mathrm{m}$ $\left(26 \mathrm{~cm}^{-1}\right)$, and $0.10 \mu \mathrm{m}\left(19 \mathrm{~cm}^{-1}\right)$, respectively, assuming that the $6.85 \mu \mathrm{m}$ feature is partially filled in by emission features (because of $\mathrm{H}_{2}$ and [Ar II]; see Lutz et al. 1996). Integrated intensities for the astronomical data (Sgr $\mathrm{A}^{*}$ ) are determined using the equation $\tau_{\text {int }}=\int \tau_{v} d v$ and are listed in Table 4.

Integrated intensity ratios predicted for (branched) saturated aliphatic hydrocarbons are well matched by those calculated from our Sgr A* spectrum (Table 4). Hydrogenated amorphous carbon (HAC) material can contain a substantial fraction of saturated aliphatic hydrocarbon groups; in fact, a $\mathrm{HAC}$ analog with $\mathrm{H} / \mathrm{C}=0.5$ and $s p^{3}$ / $s p^{2}=0.5$ provides a reasonable match to the relative strength of the 3.4, 6.85, and 7.25 $\mu \mathrm{m}$ features in the Sgr A* spectrum (Fig. 13; Furton et al. 1999).

Protostars also show a prominent feature at $6.85 \mu \mathrm{m}$ that appears to be associated with carriers residing in ices along
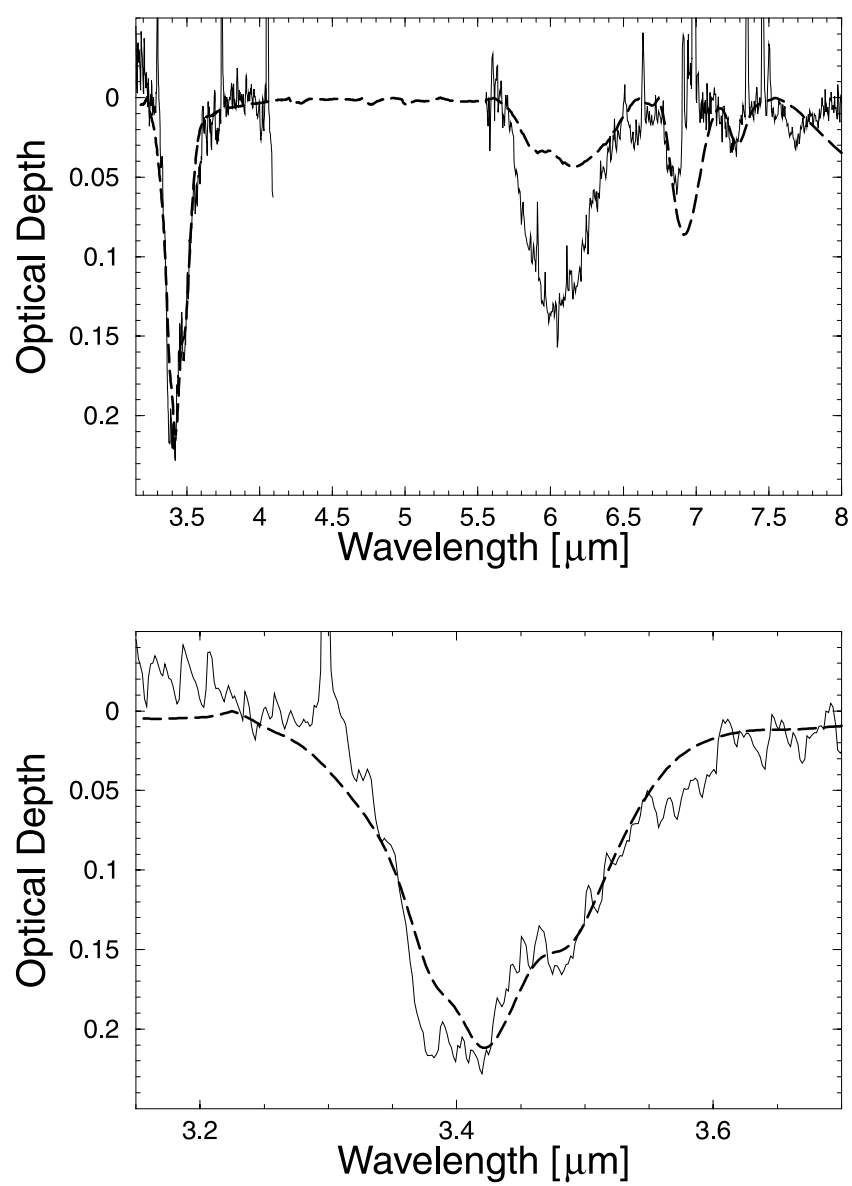

FIG. 13.-Mid-IR spectrum of Sgr A* compared with laboratory HAC (dashed line; from Furton et al. 1999. The bottom panel shows a close-up of the $3.4 \mu \mathrm{m}$ feature compared with HAC).

the line of sight (Keane et al. 2000). The $6.85 \mu \mathrm{m}$ ice band, however, extends from approximately 6.5 to $7.2 \mu \mathrm{m}$, which is much broader than that observed toward Sgr A*, GCS 3, and GCS 4 (Fig. 6). The ice profile varies in peak wavelength, width, and depth for different protostellar objects and also correlates with the $3 \mu \mathrm{m}$ ice feature (Keane et al. 2000), whereas no such strong variation in the feature is observed toward the GC, even though there are clearly different amounts of molecular cloud ices. Moreover, the 6.85 $\mu \mathrm{m}$ optical depth is very similar in the three Galactic center sources despite the large differences in ice optical depth. Therefore, we attribute the $6.85 \mu \mathrm{m}$ feature to dust in the

TABLE 4

Aliphatic Hydrocarbon Bands: SGr A*

\begin{tabular}{|c|c|c|}
\hline Band $(\mu \mathrm{m})$ & $\tau\left(\lambda_{0}\right)$ & Integrated Area $\left(\mathrm{cm}^{-1}\right)$ \\
\hline $3.4\left(\operatorname{Sgr} A^{*}\right) \ldots$ & $0.21 \pm 0.01$ & 29 \\
\hline $6.85\left(\operatorname{Sgr} A^{*}\right) \ldots \ldots \ldots \ldots \ldots$ & $0.05 \pm 0.01$ & 1.6 \\
\hline $7.25($ Sgr A*) $\ldots \ldots \ldots \ldots \ldots \ldots$ & $0.03 \pm 0.01$ & 0.7 \\
\hline Relative Integrated Intensity & Observed & Expected $^{a}$ \\
\hline$A(6.85 \mu \mathrm{m}) / A(7.25 \mu \mathrm{m})$ & 2.3 & 2.5 (branched), 5 (normal) \\
\hline$A(6.85 \mu \mathrm{m}) / A(3.4 \mu \mathrm{m}) \ldots \ldots \ldots$ & 0.06 & 0.07 \\
\hline$A(7.25 \mu \mathrm{m}) / A(3.4 \mu \mathrm{m}) \ldots \ldots \ldots$ & 0.02 & 0.03 (branched), 0.02 (normal) \\
\hline
\end{tabular}

${ }^{a}$ Expected integrated absorption area intensity for saturated aliphatic hydrocarbons from Wexler 1967. 
TABLE 5

Aromatic Hydrocarbon BANDS: GCS $3^{\mathrm{a}}$

\begin{tabular}{cccc}
\hline \hline $\begin{array}{c}\text { Band } \\
(\mu \mathrm{m})\end{array}$ & $\tau\left(\lambda_{0}\right)$ & $\begin{array}{r}\text { FWHM } \\
\left(\mathrm{cm}^{-1}\right)\end{array}$ & $\begin{array}{c}\text { Integrated Area } \\
\left(\mathrm{cm}^{-1}\right)\end{array}$ \\
\hline $3.28 \ldots \ldots$ & $0.026 \pm 0.005$ & $25 \pm 5$ & $0.8 \pm 0.3$ \\
$6.2 \ldots \ldots$. & $0.10 \pm 0.02$ & $34 \pm 5$ & $3.6 \pm 0.6$ \\
\hline
\end{tabular}

${ }^{a}$ Uncertainty does not reflect uncertainties in continuum choice.

diffuse interstellar medium, related to the $3.4 \mu \mathrm{m}$ aliphatic hydrocarbon feature, rather than molecular cloud ices.

\subsection{Aromatic Hydrocarbon Features \\ 5.2.1. The $6.2 \mu \mathrm{m}$ Band}

All the GC sources show evidence for the presence of a $6.2 \mu \mathrm{m}\left(\mathrm{FWHM}=34 \pm 5 \mathrm{~cm}^{-1}\right)$ feature (Tables 2 and 5). This feature has been detected previously in ISO spectra of GC sources and of four Wolf-Rayet stars and is attributed to the $\mathrm{C}-\mathrm{C}$ stretch in aromatic hydrocarbons (Schutte et al. 1998).

The $6.2 \mu \mathrm{m}$ profiles presented here were determined by first finding a satisfactory fit to the 3 and $6 \mu \mathrm{m}$ ice features as described in $\S 4.1$, then subtracting this "fit" from the 6 $\mu \mathrm{m}$ absorption feature to obtain a residual spectrum (Figs. $7,8,9)$. The $6.2 \mu \mathrm{m}$ profile for WR 118 is used as a template "diffuse ISM" spectrum since this line of sight is known to contain no molecular cloud material based on nondetections of typical molecular cloud features $\left(\mathrm{H}_{2} \mathrm{O}\right.$ ice and $\mathrm{CO}_{2}$ : van der Hucht et al. 1996; Schutte et al. 1998). In the cases of GCS 3 and GCS 4, this procedure leads to a residual that closely matches the (scaled) WR $1186.2 \mu \mathrm{m}$ profile. Figure 8 (right panels) shows the result of adding the scaled WR 118 profile to the ice fits for these sources and shows that the 3 and $6 \mu \mathrm{m}$ profiles can be accounted for fully by ices plus a diffuse ISM component. In the case of Sgr A*, only the red side of the residual is accounted for by a diffuse ISM (WR 118-like) component. The excellent match of the red wing of the profiles gives us confidence that Sgr A* does contain the same $6.2 \mu \mathrm{m}$ feature seen in GCS 3, GCS 4, and the WR stars.

The $6.2 \mu \mathrm{m}$ feature is most likely carried by dust unassociated with molecular cloud material since it is detected in lines of sight known to contain no ices (such as $\mathrm{H}_{2} \mathrm{O}, \mathrm{CO}_{2}$; Schutte et al. 1998). In the Galactic center, the source with the strongest ice bands (Sgr A*) has the smallest $6.2 \mu \mathrm{m}$ feature. For all the sources studied, there appears to be a trend of increasing $6.2 \mu \mathrm{m}$ feature depth with $A_{V}$ and $\tau_{9.7}$ (Schutte et al. 1998); however, the depth of the $6.2 \mu \mathrm{m}$ feature in the Sgr A* spectrum seems to be anomalously weak with respect to its $9.7 \mu \mathrm{m}$ silicate optical depth and its extinction in comparison with sources in the solar neighborhood (Schutte et al. 1998) and with GCS 3 and GCS 4. A depth of $\tau_{6.2} \sim 0.13$ would be expected for Sgr A* if this quantity was correlated with the silicate depth. In contrast, the observed depth is only 0.05 (Table 2). Hence, it seems that the $6.2 \mu \mathrm{m}$ feature is carried by an independent component the abundance of which varies with respect to that of the silicates. We note that the total visual extinction $\left(A_{V}\right)$ also seems to vary independently of the $9.7 \mu \mathrm{m}$ silicate band (Roche \& Aitken 1985).

The presence of aromatic hydrocarbons can also be determined from observations of the complimentary $3.3 \mu \mathrm{m}$ $\mathrm{C}-\mathrm{H}$ stretch feature. This feature is discussed in more detail below.

\subsubsection{The $3.28 \mu \mathrm{m}$ Aromatic Hydrocarbon Feature}

The AOT6 spectrum of GCS 3 exhibits a weak absorption feature centered at $3.28 \mu \mathrm{m}$, with $\Delta v=25 \pm 5 \mathrm{~cm}^{-1}$, which we attribute to the $\mathrm{C}-\mathrm{H}$ stretch in aromatic hydrocarbons (Fig. 12, Table 5). The $\mathrm{C}-\mathrm{H}$ stretch in solid methane occurs near $3.32 \mu \mathrm{m}$, far from the observed feature, therefore we can rule out methane as a possible candidate for the absorption. The lower resolution/signal-to-noise AOT1 spectrum is also consistent with the presence of absorption centered at $3.28 \mu \mathrm{m}$. Presence of this feature in the AOT1 spectrum of GCS 4 cannot be ruled out. An absorption feature centered at $\lambda_{0} \sim 3.25 \mu \mathrm{m}(\Delta v=74$

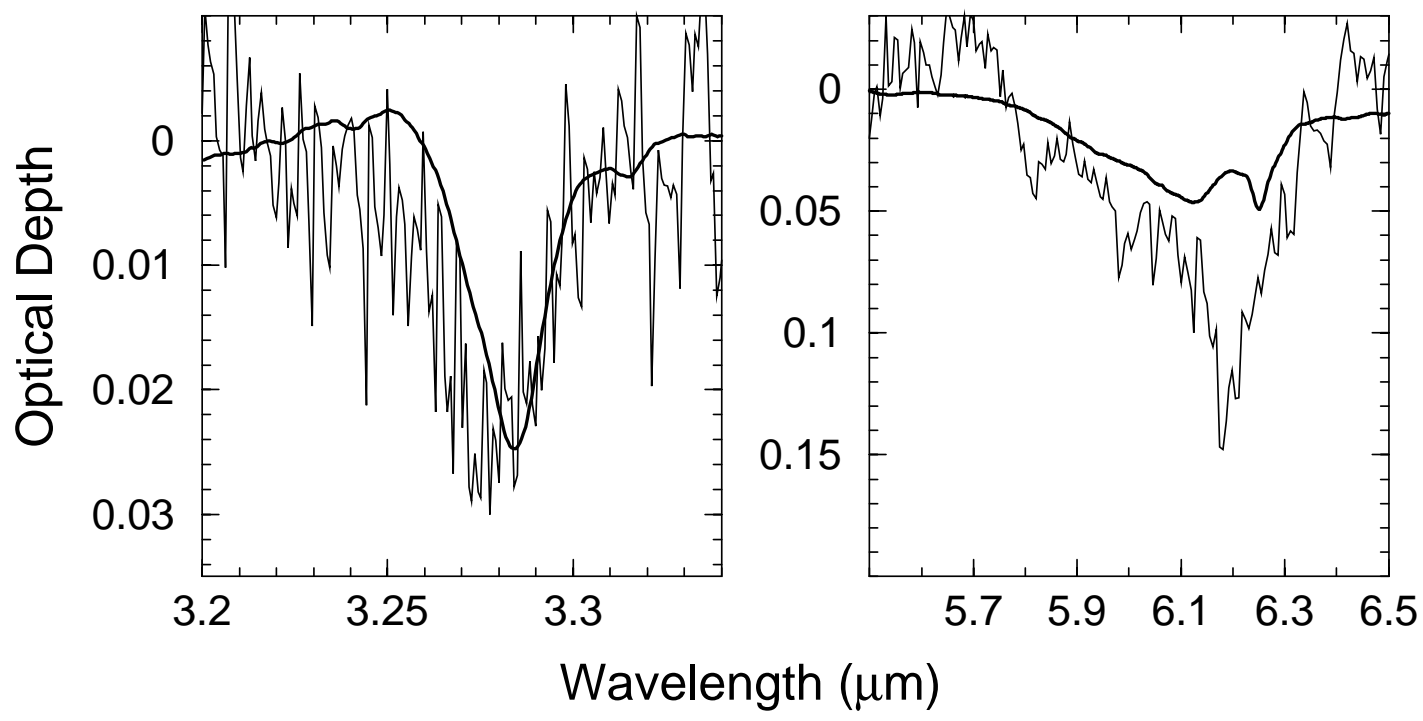

FIG. 14.-Aromatic $\mathrm{C}-\mathrm{H}$ stretch (left) and $\mathrm{C}-\mathrm{C}$ stretch (right) spectral regions in GCS 3 compared with a $\mathrm{H}_{2} \mathrm{O}: \mathrm{C}_{16} \mathrm{H}_{10}(14 \mathrm{~K})$ laboratory mixture (smooth solid line). The $6.2 \mu \mathrm{m}$ band in the laboratory mixture is scaled based on the strength of the $3.28 \mu \mathrm{m}$ band. It is apparent that a PAH in ice mixture cannot account for most of the observed $6.2 \mu \mathrm{m}$ absorption. Spectra shown are AOT6. 
$\mathrm{cm}^{-1}$ ) has been detected in molecular cloud sources and is attributed to aromatic hydrocarbon molecules at low temperature (Sellgren et al. 1995; Brooke et al. 1999). The difference in central wavelength and width between these different environments cannot be accounted for by uncertainties in the continuum fit but, rather, demonstrates the difference in the temperature and/or carrier of the aromatic material in these regions. Although there is a strong $\operatorname{Pf} \delta$ atomic hydrogen line at $3.30 \mu \mathrm{m}$, there is no evidence for an underlying absorption feature at $3.28 \mu \mathrm{m}$, to a limit of $\tau_{3.28}<0.02$, in the Sgr A* spectrum. This limit is consistent with the weakness of the $6.2 \mu \mathrm{m}$ aromatic band in Sgr A*, thus we conclude that the 3.28 and $6.2 \mu \mathrm{m}$ features are caused by the same material.

Gas-phase PAHs are not likely carriers of the $3.28 \mu \mathrm{m}$ absorption feature: based on the temperature dependence of the gas-phase PAH profile determined in the laboratory by Joblin et al. (1995), a temperature of $600-1300 \mathrm{~K}$ is implied for the feature observed toward GCS 3. While such a high temperature is consistent with PAH emissionindeed $3.28 \mu \mathrm{m}$ is also the position of the unidentified infrared emission feature - to get a PAH absorption feature would require an extraordinarily large column of hot gas. Comparison of the peak positions of matrix-isolated laboratory PAHs given by Hudgins \& Sandford (1998a,1998b) with our astronomical data show that neutral PAHs, such as pyrene, frozen in ices may be a good candidate for the observed feature. Laboratory studies carried out in the Astrophysics Laboratory at NASA/Ames of pyrene in $\mathrm{H}_{2} \mathrm{O}$ ice show a fair match to the $3.28 \mu \mathrm{m}$ feature (Fig. 14). However, this does not provide simultaneously a good fit to the observed interstellar $6.2 \mu \mathrm{m}$ band (cf. $\S 4.1 .2$, Fig. 14). In fact, as pointed out by Schutte et al. (1998), neutral PAHs have too weak an absorption feature to account for the observed strength of the $6.2 \mu \mathrm{m}$ feature. For that reason, Schutte et al. (1998) attributed the $6.2 \mu \mathrm{m}$ band to ionized PAHs the CC modes of which are intrinsically much stronger. Nevertheless, this assignment still requires $10 \%$ of the available carbon in the form of PAH cations for GCS 3 and GCS 4.

An origin of the $3.28 \mu \mathrm{m}$ band in HACs is also possible. HACs, when heated, lose much of their hydrogen and their aromatic component grows in strength. The spectral characteristics of heat-treated HACs (Scott \& Duley 1996; Blanco et al. 1988) resemble those observed toward GCS 3. At present, we cannot distinguish observationally whether free-flying, ionized PAHs or HACs are the carriers of the $3.28 \mu \mathrm{m}$ band in the diffuse ISM.

\section{ORIGIN AND EVOLUTION OF ALIPHATIC AND AROMATIC HYDROCARBON DUST}

Whereas the distribution of aliphatic hydrocarbon dust, detected via the $3.4,6.85$, and $7.25 \mu \mathrm{m}$ features, toward the GC shows little variation, aromatic hydrocarbons identified via their $\mathrm{C}-\mathrm{H}$ stretch $(3.28 \mu \mathrm{m})$ and $\mathrm{C}-\mathrm{C}$ stretch $(6.2 \mu \mathrm{m})$, however, may not be evenly distributed throughout the GC region $(\$ 5.2)$. It has been proposed recently that the Quintuplet sources are dusty late-type WC stars. WC stars are descendants of massive $\left(M>25 M_{\odot}\right)$ OB stars where substantial mass loss and mixing have uncovered freshly synthesized material at the stellar surface. These objects are rich in $\mathrm{He}, \mathrm{C}$, and $\mathrm{O}(\simeq 10: 3: 1$ by number $)$, but there is no evidence for $\mathrm{H}$ or $\mathrm{N}\left(<10^{-2}\right.$ and $10^{-3}$ relative to $\mathrm{He}$ by number; Willis 1982; Nugis 1982; Torres 1988). Thus, aro- matic dust associated with the $6.2 \mu \mathrm{m} \mathrm{C}-\mathrm{C}$ stretch feature, detected toward GCS 3, GCS 4, and the well-known WR stars, may be associated with the environment of these objects instead of the general diffuse ISM. One of the IR sources (IRS 6E) included in the ISO-SWS beam centered on $\mathrm{Sgr} \mathrm{A}^{*}$ has been identified as a late-type WC star (Krabbe et al. 1995); this may explain the presence of a small amount of $6.2 \mu \mathrm{m}$ absorption (relative to its deep 9.7 $\mu \mathrm{m}$ silicate feature). However, while the WC stars may be responsible for producing the carrier(s) of the $\mathrm{C}-\mathrm{C}$ stretch $(6.2 \mu \mathrm{m})$, they cannot be a significant contributor to the carrier(s) of the $\mathrm{C}-\mathrm{H}$ stretch $(3.28 \mu \mathrm{m})$. At present, the connection between 3.28 and $6.2 \mu \mathrm{m}$ absorption features is not well established observationally.

Various models have been proposed for the origin and evolution of these dust components-ices, aliphatic, and aromatic dust - in the ISM. Of particular relevance are the processing of interstellar ices leading to organic grains (Greenberg 1989) and the processing of hydrogenated amorphous carbon (Scott \& Duley 1996; Blanco et al. 1988). Ices are generally thought to form by accretion on preexisting silicate cores inside dense molecular clouds. These ices can be photolyzed by penetrating UV photons and may be converted into an organic refractory mantle. Laboratory studies have shown that this process may lead to a material with IR characteristics matching the observed interstellar $3.4 \mu \mathrm{m}$ bands well (Greenberg et al. 1995; Allamandola, Sandford, \& Valero 1988). However, such residues invariably show much stronger absorption in the 5-8 $\mu \mathrm{m}$ range because of residual oxygen bonded to the carbon structures. Such bands are not observed in the spectra of sources showing the $3.4 \mu \mathrm{m}$ feature (Figs. 4,11 ). Our results thus strengthen the argument (Chiar et al. 1998b; Adamson et al. 1999; see $\S 1$ ) that the $3.4 \mu \mathrm{m}$ carrier is not an organic refractory mantle produced by irradiation of ices in molecular clouds.

Alternatively, HAC, which in structure is akin to soot, could be an important stardust component injected into the ISM by low-mass stars during the AGB phase of their evolution, in agreement with the strength of the $3.4 \mu \mathrm{m}$ absorption band in the protoplanetary object CRL 618 (Chiar et al. 1998b). Extensive analysis of interstellar observations require that the amount of $\mathrm{C}$ locked up in $\mathrm{HAC}$ relative to $\mathrm{H}$ nuclei is $\sim 8 \times 10^{-5}$ (Furton et al. 1999). This depends on the extent to which the carbonaceous material is hydrogenated, but the adopted value $\mathrm{H} / \mathrm{C} \sim 0.5$ is most consistent with other HAC properties (Furton et al. 1999). It has been suggested that HAC could also be formed through accretionary processes in the diffuse ISM but plausible chemical mechanisms have not been identified (Duley \& Williams 1995). Nevertheless, HACs are thought to evolve in the diffuse ISM: under the influence of FUV irradiation (or heating), HACs lose $\mathrm{H}$ and their structure is transformed from an aliphatic structure into a more aromatic structure (Ogmen \& Duley 1988; Furton \& Witt 1993). This process will be counteracted by hydrogenation. In photodissociation regions, the astrophysical sites of these processes, both UV irradiation and exposure to atomic hydrogen will occur at the same time (Furton \& Witt 1993). The relative amount of aromatic and aliphatic carbon is then set by the balance between these two processes and this will determine the relative strength of the 3.3 (and 6.2) $\mu \mathrm{m}$ band compared with the $3.4 \mu \mathrm{m}$ band. We note here that, if the 3.3 and 6.2 bands observed in GCS 3 are associated with 
the diffuse ISM rather than circumstellar dust, we may actually be observing both the aromatic and aliphatic components involved in this process along these lines of sight. However, models of this type have problems too. In particular, theoretical studies of dust destruction and observational studies of elemental depletions suggest that the lifetime of stardust is much shorter $\left(3 \times 10^{8} \mathrm{yr}\right)$ than the injection timescale by old stars $\left(5 \times 10^{9} \mathrm{yr}\right)$, which suggests that dust formation in the ISM is very important (Jones et al. 1994, 1996). Now, in the models, ices accreted inside dense molecular clouds, would be rapidly destroyed when the molecular cloud is dispersed. In an evolutionary sense, ices would have little influence on the dust composition in the diffuse ISM. However, it has been suggested that organic residues produced by UV photolysis of interstellar ices are rapidly carbonized ( $\mathrm{H}, \mathrm{O}$, and $\mathrm{N}$ are lost) by the strong far-UV fields in the diffuse ISM and that this leads to a HAC-like mantle (Jenniskens et al. 1993). It remains puzzling then that, while interstellar ice, as well as silicate, features can be polarized, the $3.4 \mu \mathrm{m}$ aliphatic hydrocarbon feature is not (Adamson et al. 1999). Possibly, this might imply a link between the aliphatic hydrocarbon carriers of the $3.4 \mu \mathrm{m}$ feature and the aromatic carrier of the $2175 \AA$ bump that is also not polarized (Martin et al. 1999). In any case, an HAC-like material with very few oxygen impurities, is the carrier of the $3.4,6.85$, and $7.25 \mu \mathrm{m}$ features. Further observations, particularly of the $3.28,3.4$, and $6.2 \mu \mathrm{m}$ bands will be instrumental in settling the origin and evolution of this component.

\section{SUMMARY}

We have identified molecular cloud and diffuse interstellar medium material along the line of sight to the Galactic center using ISO-SWS data for three sight lines. The amount of molecular cloud material varies across the GC field, and the amount of diffuse ISM material shows little variation. This is supported by our observations of the $3 \mu \mathrm{m}$ $\mathrm{H}_{2} \mathrm{O}$ ice feature that ranges between $\tau_{3.0}=0.16$ and $\tau_{3.0}=$ 0.50 , and the $3.4 \mu \mathrm{m}$ aliphatic hydrocarbon feature that ranges between $\tau_{3.4}=0.15$ and $\tau_{3.4}=0.21$. The line of sight toward Sgr A* contains the largest column of molecular cloud material, relative to GCS 3 and GCS 4, apparent from the depth of the $3 \mu \mathrm{m}$ ice feature. Toward all GC sources discussed in this paper, $\mathrm{CO}_{2}$ ice is detected, and $\mathrm{CO}$ is present mainly in the gas phase. Toward Sgr $\mathrm{A}^{*}$, solid $\mathrm{CH}_{4}$ has also been clearly identified. As the amount of solid $\mathrm{CO}_{2}$ relative to $\mathrm{H}_{2} \mathrm{O}$ is higher toward the Quintuplet sources in comparison to that toward Sgr A*, there may be more than one molecular cloud associated with the overall GC region. However, the processes that control the solid $\mathrm{CO}_{2}$ abundance are not well understood (e.g., Whittet et al. 1998; Gerakines et al. 1999).

In many ways, the molecular clouds along the line of sight toward the Galactic center are similar to local molecular clouds in terms of abundances of key solid state molecules (Table 6). Water-ice remains the most abundant solid state molecule; the solid $\mathrm{CO}_{2} / \mathrm{H}_{2} \mathrm{O}$ ratio is within the range found for local molecular clouds (Gerakines et al. 1999); $\mathrm{CH}_{3} \mathrm{OH} / \mathrm{H}_{2} \mathrm{O}$ is similar to that observed in quiescent clouds such as Taurus (Chiar et al. 1996) and does not resemble the higher abundance observed toward heavily embedded massive protostars. While solid $\mathrm{CO}$ is not detected in the sight lines discussed here, a weak $4.67 \mu \mathrm{m}$
TABLE 6

Comparison of Ice Abundances toward SagitTarius A* AND NGC 7538 IRS 9a

\begin{tabular}{|c|c|c|c|}
\hline Molecule & Sgr $A^{*}$ & NGC 7538 IRS 9 & References $^{b}$ \\
\hline $\mathrm{H}_{2} \mathrm{O} \ldots \ldots \ldots \ldots$ & 100 & 100 & 1 \\
\hline $\mathrm{NH}_{3} \ldots \ldots \ldots \ldots$ & $20-30$ & 10 & 2 \\
\hline $\mathrm{CO}_{2} \ldots \ldots \ldots$ & 14 & 20 & 3 \\
\hline $\mathrm{CO} \ldots \ldots \ldots \ldots$ & $<12$ & 15 & 4 \\
\hline $\mathrm{CH}_{3} \mathrm{OH} \ldots \ldots$. & $<4$ & 7 & 5 \\
\hline $\mathrm{CH}_{4} \ldots \ldots \ldots \ldots$ & 2 & 2 & 6 \\
\hline НСООН...... & 6 & 3 & 7 \\
\hline
\end{tabular}

\footnotetext{
${ }^{\text {a }}$ Abundances are percentages relative to $\mathrm{H}_{2} \mathrm{O}$ ice.

${ }^{b}$ References are for NGC 7538 IRS 9 ice column densities. Column densities for Sgr $\mathrm{A}^{*}$ are from this paper.

REFERENCES. - (1) Tielens et al. 1991; (2) Lacy et al. 1998; (3) Gerakines et al. 1999; (4) Chiar et al. 1998a; (5) Dartois et al. 1999; (6) Boogert et al. 1998; (7) Schutte et al. 1999.
}

feature has been detected previously (McFadzean et al. 1989). The $\mathrm{CO} / \mathrm{H}_{2} \mathrm{O}$ ratio implied is less than $10 \%$. The 3.0 $\mu \mathrm{m}$ ice profile is indicative of the temperature as well as the content of the ices. The profile observed toward the GC is distinct from that seen in local molecular clouds such as Taurus. It is consistent with cold $(\sim 15 \mathrm{~K})$ ices, similar to local quiescent dense clouds, but setting this feature apart from those studied in local clouds is substructure at $2.95 \mu \mathrm{m}$ indicative of a relatively high abundance (up to $\sim 30 \%$, relative to $\mathrm{H}_{2} \mathrm{O}$ ) of $\mathrm{NH}_{3}$ in the ices. Toward local molecular cloud sources, the solid $\mathrm{NH}_{3} / \mathrm{H}_{2} \mathrm{O}$ abundance is observed to be $\sim 10 \%$ (e.g., Whittet et al. 1996, Lacy et al. 1998). The high ammonia abundance in GC molecular clouds could reflect the higher N/O ratio in the inner Galaxy.

Aliphatic hydrocarbons, first detected via their $3.4 \mu \mathrm{m}$ $\mathrm{CH}$ stretch feature, are a widespread component of the diffuse interstellar medium. ISO-SWS has detected for the first time the corresponding $\mathrm{C}-\mathrm{H}$ deformation modes at 6.85 and $7.25 \mu \mathrm{m}$ in the line of sight toward the Galactic center. The ratio of integrated intensities for these three absorption features are consistent with the predicted values for saturated aliphatic hydrocarbons (Wexler 1967). Hydrogenated amorphous carbon containing a substantial fraction of saturated aliphatic hydrocarbon groups provides a convincing match to the observed hydrocarbon absorption features and is the likely carrier of these features.

Aromatic hydrocarbons, detected via their $3.3 \mu \mathrm{m} \mathrm{C}-\mathrm{H}$ and $6.2 \mu \mathrm{m} \mathrm{C}-\mathrm{C}$ stretching vibrations, do not appear to be evenly distributed throughout the Galactic center. Absorption at $3.28 \mu \mathrm{m}$ is detected in the line of sight toward GCS 3 but not toward Sgr A*. In addition, the $6.2 \mu \mathrm{m}$ absorption feature toward Sgr A* is much weaker than that toward the Quintuplet sources. Although our results suggest that the 3.28 and $6.2 \mu \mathrm{m}$ features are caused by the same carrier, it remains unclear whether the $\mathrm{C}-\mathrm{C}$ stretch absorption feature could instead be produced by dusty C-rich WR stars and is thus independent of the $\mathrm{C}-\mathrm{H}$ stretch feature.

J. E. C. was supported by a National Research Council associateship for a portion of this work and is currently supported by NASA's Long-Term Space Astrophysics program under grant 399-20-61-02. D. C. B. W. is supported by NASA through grants NAG5-7598, NAG5-7884, and JPL contract 961624. 


\section{REFERENCES}

Adamson, A. J., Whittet, D. C. B., Chrysostomou, A., Hough, J. H., Aitken, D. K., Wright, G. S., \& Roche, P. F. 1999, ApJ, 512, 224

Aitken, D. K., Briggs, G. P., Roche, P. F., Bailey, J. A., \& Hough, J. H. 1986, MNRAS, 218, 363

Allamandola, L. J., Sandford, S. A., Tielens, A. G. G. M., \& Herbst, T. M. 1992, ApJ, 399, 134

Allamandola, L. J., Sandford, S. A., \& Valero, G. J. 1988, Icarus, 76, 225

Becklin, E. E., Matthews, K., Neugebauer, G., \& Willner, S. P. 1978, ApJ, 219,121

Blanco, A., Bussoletti, E., \& Colangeli, L. 1988, ApJ, 334, 875

Boogert, A. C. A., Helmich, F. P., van Dishoeck, E. F., Schutte, W. A., Tielens, A. G. G. M., \& Whittet, D. C. B. 1998, A\&A, 336, 352

Boogert, A. C. A., Schutte, W. A., Helmich, F. P., Tielens, A. G. G. M., \& Wooden, D. H. 1997, A\&A, 317, 929

Boogert, A. C. A., et al. 1996, A\&A, 315, L377

Bridger, A., Wright, G., \& Geballe, T. 1993, in Conference Abstract Volume of Infrared Astronomy with Arrays: The Next Generation, ed. I. McLean (Los Angeles: UCLA Dept. of Astronomy), 537

Brooke, T. Y., Sellgren, K., \& Geballe, T. R. 1999, ApJ, 517, 883

Butchart, I., McFadzean, A. D., Whittet, D. C. B., Geballe, T. R., \& Greenberg, J. M. 1986, A\&A, 154, L5

Chiar, J. E., Adamson, A. J., \& Whittet, D. C. B. 1996, ApJ, 472, 665

Chiar, J. E., Gerakines, P. A., Whittet, D. C. B., Pendleton, Y. J., Tielens, A. G. G. M., \& Boogert, A. C. A. 1998a, ApJ, 498, 716

Chiar, J. E., Pendleton, Y. J., Geballe, T. G., \& Tielens, A. G. G. M. 1998b, ApJ, 507, 281

Dartois, E., Schutte, W., Geballe, T. R., Demyk, K., Ehrenfreund, P., \& d'Hendecourt, L. 1999, A\&A, 342, L32

de Graauw, T., et al. 1996a, A\&A, 315, L49

1996b, A\&A, 315, L345

d'Hendecourt, L., et al. 1996, A\&A, 315, L365

Duley, W. W., Scott, A. D., Seahra, S., \& Dadswell, G. 1998, ApJ, 503, L183

Duley, W. W., \& Williams, D. A. 1995, MNRAS, 272, 442

Eckart, A., Genzel, R., Hofmann, R., Sams, B. J., \& Tacconi-Garman, L. E. 1995, ApJ, 445, L23

Figer, D. F., McLean, I. S., \& Morris, M. 1999, ApJ, 514, 202

Furton, D. G., Laiho, J. W., \& Witt, A. N. 1999, ApJ, 526, 752

Furton, D. G., \& Witt, A. N. 1993, ApJ, 415, L51

Gerakines, P. A., Schutte, W. A., \& Ehrenfreund, P. 1996, A\&A, 312, 289

Gerakines, P. A., Schutte, W. A., Greenberg, J. M., \& van Dishoeck, E. F. 1995, A\&A, 296, 810

Gerakines, P. A., et al. 1999, ApJ, 522, 357

Giard, M., Serra, G., Caux, E., Pajot, F., \& Lamarre, J. M. 1988, A\&A, 201, L1

Gibb, E. L., et al. 2000, ApJ, 536, 347

Greenberg, J. M. 1989, in IAU Symp. 135, Interstellar Dust, ed. L. J. Allamandola \& A. G. G. M. Tielens (Dordrecht: Reidel), 345

Greenberg, J. M., Li, A., Mendoza-Gomez, C. X., Schutte, W. A., Gerakines, P. A., \& De Groot, M. 1995, ApJ, 455, L177

Hagen, W., \& Tielens, A. G. G. M., \& Greenberg, J. M. 1981, Chem. Phys., 56,367

Hudgins, D. M., \& Sandford, S. A. 1998a, J. Phys. Chem. A, 102, 329 1998b, J. Phys. Chem. A, 102, 344

Hudgins, D. M., Sandford, S. A., Allamandola, L. J., \& Tielens, A. G. G. M. 1993, ApJS, 86, 713

Jenniskens, P., Baratta, G. A., Kouchi, A., De Groot, M. S., Greenberg, J. M., \& Strazzulla, G. 1993, A\&A, 273, 583

Joblin, C., Boissel, P., Leger, A., D'Hendecourt, L., \& Defourneau, D. 1995, A\&A, 299, 835

Jones, A. P., Tielens, A. G. G. M., \& Hollenbach, D. J. 1996, ApJ, 469, 740

Jones, A. P., Tielens, A. G. G. M., Hollenbach, D. J., \& McKee, C. F. 1994, ApJ, 433, 797

Keane, J. V., Tielens, A. G. G. M., Boogert, A. C. A., Schutte, W. A., \& Whittet, D. C. B. 2000, A\&A, submitted

Kobayashi, Y., Okuda, H., Sato, S., Jugaku, J., \& Dyck, H. M. 1983, PASJ, 35,101

Krabbe, A., et al. 1995, ApJ, 447, L95

Lacy, J. H., Faraji, H., Sandford, S. A., \& Allamandola, L. J. 1998, ApJ, 501, L105

Lebofsky, M. J., Rieke, G. H., \& Tokunaga, A. T. 1982, ApJ, 263, 736
Lequeux, J., \& Jourdain de Muizon, M. 1990, A\&A, 240, L19

Lutz, D., et al. 1996, A\&A, 315, L269

Maréchal, Y. 1987, J. Chem. Phys., 87, 6344

Martin, P. G., Clayton, G. C., \& Wolff, M. J. 1999, ApJ, 510, 905

Mattila, K., Lemke, D., Haikala, L. K., Laureijs, R. J., Leger, A., Lehtinen, K., Leinert, C., \& Mezger, P. G. 1996, A\&A, 315, L353

McFadzean, A. D., Whittet, D. C. B., Bode, M. F., Adamson, A. J., \& Longmore, A. J. 1989, MNRAS, 241, 873

McKee, C. F. 1989, ApJ, 345, 782

Moneti, A., \& Cernicharo, J. 2000, ApJ, submitted

Moneti, A., Glass, I., \& Moorwood, A. 1992, MNRAS, 258, 705

1994, MNRAS, 268, 194

Moore, M. H., Ferrante, R. F., \& Nuth, J. A. I. 1996, Planet. Space Sci., 44 927

Nagata, T., Woodward, C. E., Shure, M., Pipher, J. L., \& Okuda, H. 1990, ApJ, 351, 83

Nugis, T. 1982, in Wolf Rayet Stars: Observations, Physics, Evolution, ed. C. W. H. de Loore \& A. J. Willis (Dordrecht: Kluwer), 131

Ogmen, M., \& Duley, W. W. 1988, ApJ, 334, L117

Okuda, H., et al. 1990, ApJ, 351, 89

Pendleton, Y. J., Sandford, S. A., Allamandola, L. J., Tielens, A. G. G. M., \& Sellgren, K. 1994, ApJ, 437, 683

Pendleton, Y. J., Tielens, A. G. G. M., \& Werner, M. W. 1990, ApJ, 349, 107

Rieke, G. H., Rieke, M. J., \& Paul, A. E. 1989, ApJ, 336, 752

Ristorcelli, I., Giard, M., Meny, C., Serra, G., Lamarre, J. M., Le Naour, C., Leotin, J., \& Pajot, F. 1994, A\&A, 286, L23

Roche, P. F., \& Aitken, D. K. 1985, MNRAS, 215, 425

Rubin, R. H., Simpson, J. P., Erickson, E. F., \& Haas, M. R. 1988, ApJ, 327, 377

Sandford, S. A., Allamandola, L. J., Tielens, A., Sellgren, K., Tapia, M., \& Pendleton, Y. 1991, ApJ, 371, 607

Schutte, W. A., et al. 1999, A\&A, 343, 966

Schutte, W. A., Gerakines, P. A., Geballe, T. R., van Dishoeck, E. F., \& Greenberg, J. M. 1996a, A\&A, 309, 633

Schutte, W. A., \& Greenberg, J. M. 1989, in Dust in the Universe, ed. M. E. Bailey \& D. A. Williams (Cambridge: Cambridge Univ. Press), 403

Schutte, W. A., et al. 1996b, A\&A, 315, L333

1998, A\&A, 337, 261

Scott, A., \& Duley, W. W. 1996, ApJ, 472, L123

Sellgren, K., Brooke, T. Y., Smith, R. G., \& Geballe, T. R. 1995, ApJ, 449, L69

Sellgren, K., Hall, D. N. B., Kleinmann, S. G., \& Scoville, N. Z. 1987, ApJ, 317,881

Simpson, J. P., Colgan, S. W. J., Rubin, R. H., Erickson, E. F., \& Haas, M. R. 1995, ApJ, 444, 721

Skinner, C. J., Tielens, A. G. G. M., Barlow, M. J., \& Justtanont, K. 1992, ApJ, 399, L79

Smith, R. G., Sellgren, K., \& Tokunaga, A. T. 1989, ApJ, 344, 413

Strazzulla, G., Castorina, A. C., \& Palumbo, M. E. 1995, Planet. Space Sci., 43,1247

Tielens, A. G. G. M., \& Allamandola, L. J. 1987, in Interstellar Processes, ed. D. Hollenbach \& H. Thronson (Dordrecht: Kluwer), 397

Tielens, A. G. G. M., Tokunaga, A. T., Geballe, T. R., \& Baas, F. 1991, ApJ, 381,181

Tielens, A. G. G. M., Wooden, D. H., Allamandola, L. J., Bregman, J., \& Witteborn, F. C. 1996, ApJ, 461, 210

Torres, A. V. 1988, ApJ, 325, 759

van der Hucht, K. A., et al. 1996, A\&A, 315, L193

Vriend, W. J. 2000, Master's thesis, Univ. of Groningen

Wada, S., Sakata, A., \& Tokunaga, A. T. 1991, ApJ, 375, L17

Wexler, A. S. 1967, Appl. Spectrosc. Rev., 1, 29

Whittet, D. C. B., et al. 1997, ApJ, 490, 729

1998, ApJ, 498, L159 1996, ApJ, 458, 363

Willis, A. J. 1982, in Wolf Rayet Stars: Observations, Physics, Evolution, ed. C. W. H. de Loore \& A. J. Willis (Dordrecht: Kluwer), 87

Willner, S. P., Russell, R. W., Puetter, R. C., Soifer, B. T., \& Harvey, P. M 1979, ApJ, 229, L65

Wollman, E. R., Smith, H. A., \& Larson, H. P. 1982, ApJ, 258, 506 